\title{
NON-LINEAR MAPS ON SELF-ADJOINT OPERATORS PRESERVING NUMERICAL RADIUS AND NUMERICAL RANGE OF LIE PRODUCT
}

\author{
JINCHUAN HOU AND KAN HE
}

\begin{abstract}
Let $H$ be a complex separable Hilbert space of dimension $\geq 2, \mathcal{B}_{s}(H)$ the space of all self-adjoint operators on $H$. We give a complete classification of non-linear surjective maps on $\mathcal{B}_{s}(H)$ preserving respectively numerical radius and numerical range of Lie product.
\end{abstract}

\section{INTRODUCTION}

Let $A$ be a bounded linear operator acting on a complex Hilbert space $H$. Recall that the numerical range of $A$ is the set $W(A)=\{\langle A x, x\rangle \mid x \in H,\|x\|=1\}$, and the numerical radius of $A$ is $w(A)=\sup \{|\lambda| \mid \lambda \in W(A)\}$. The problem of characterizing linear maps on matrices or operators that preserve numerical range or numerical radius has been studied by many authors, see for example [3, 4, 17, 18] and the references therein. In recent years, interest in characterizing general (non-linear) preservers of numerical ranges or numerical radius has been growing $([1,2,15,6,18,9,11,12,13,15,16,17,19])$.

Let $\mathcal{B}_{s}(H)$ and $\mathcal{B}(H)$ the space of all self-adjoint operators and the algebra of all bounded linear operators on complex Hilbert space $H$, respectively. Suppose that $\mathcal{A}=\mathcal{B}(H)$ or $\mathcal{B}_{s}(H)$, and $F$ is the numerical range $W$ or numerical radius $w$. Let $A \circ B$ denote any product of a pair of $A, B \in \mathcal{A}$ such as operator product $A B$, Jordan product $A B+B A$, Jordan semi-triple product $A B A$ and Lie product $A B-B A$. A map $\Phi: \mathcal{A} \rightarrow \mathcal{A}$ preserves numerical range (or numerical radius) of product $\circ$ if $F=W($ or $F=w)$ and $\Phi$ satisfies

$$
F(A \circ B)=F(\Phi(A) \circ \Phi(B))
$$

for all $A, B \in \mathcal{A}$.

Assume that $\Phi: \mathcal{A} \rightarrow \mathcal{A}$ satisfy Eq.(1.1). For the case $F=W$ and $\Phi$ is surjective, it was shown in [15] that if $A \circ B=A B$ and $\mathcal{A}=\mathcal{B}(H)$, then there exists a unitary operator $U$ such that $\Phi(A)=\epsilon U A U^{*}$ for all $A \in \mathcal{A}$, where $\epsilon \in\{-1,1\}$; if $A \circ B=A B A$ and $\mathcal{A}=\mathcal{B}(H)$, then $\Phi$ is the multiple of a $\mathrm{C}^{*}$-isomorphism (by a cubic root of unity); if $A \circ B=A B$ and $\mathcal{A}=\mathcal{B}_{s}(H)$, then there exists a unitary operator $U$ such that $\Phi(A)=\epsilon U A U^{*}$ for all $A \in \mathcal{A}$, where $\epsilon \in\{-1,1\}$. For the case $F=w, A \circ B=A B, \mathcal{A}=\mathcal{B}(H)$ and $\Phi$ is surjective, it was proved in [6] that there exist a unitary or anti-unitary operator $U$ and a unit-modular functional $f: \mathcal{A} \rightarrow \mathbb{C}$ such that $\Phi(A)=f(A) U A U^{*}$ for all $A \in \mathcal{A}$. The case of $F=w, A \circ B=A B A$

2002 Mathematical Subject Classification. 47H20, 47B49, 47A12.

Key words and phrases. Numerical range, numerical radius, Lie product of operators, general preservers.

This work supported by National Science Foundation of China (11171249, 11201329,11271217) and Program for the Outstanding Innovative Teams of Higher Learning Institutions of Shanxi. 
and $\mathcal{A}=\mathcal{B}(H)$ was dealt with in [10]. For the case when $F=w, A \circ B=A B$ or $A B A$ and $\mathcal{A}=\mathcal{B}_{s}(H)$, the results obtained in [12] reveal that there is a unitary operator or conjugate unitary operator $U$ on $H$, a sign function $h: \mathcal{S}(H) \rightarrow\{1,-1\}$ such that $\Phi(T)=h(T) U T U^{*}$ for any $T \in \mathcal{B}_{s}(H)$. Maps preserving numerical range of Jordan product are characterized in [9, 11, 17].

Recent interest is focused on characterizing non-linear maps preserving numerical range or numerical radius of Lie product. When $3 \leq \operatorname{dim} H=n<\infty, \mathrm{Li}$, Poon and Sze [19] proved that a surjective map $\Phi: \mathcal{B}(H) \rightarrow \mathcal{B}(H)$ satisfies $w(\Phi(A) \Phi(B)-\Phi(B) \Phi(A))=w(A B-B A)$ for all $A, B \in \mathcal{A}$ if and only if there exists a unitary matrix $U$ such that

$$
\Phi(A)=\mu_{A} U A^{\dagger} U^{*}+\nu_{A} I
$$

for all $A \in \mathcal{A}$, where $\mu_{A}, \nu_{A} \in \mathbb{C}$ depend on $A$ with $\left|\mu_{A}\right|=1,(\cdot)^{\dagger}$ stands for one of the following four maps: $A \mapsto A, A \mapsto \bar{A}, A \mapsto A^{t}$ and $A \mapsto A^{*}$. For arbitrary dimensional space $H$ (concluding infinite and two dimensional cases), without assumption of surjectivity, Hou, Li and Qi [16] gave a characterization of maps on $\mathcal{B}(H)$ preserving numerical range of Lie product.

Theorem HLQ. Let $H, K$ be complex Hilbert spaces of dimension $\geq 2$ and $\Phi: \mathcal{B}(H) \rightarrow$ $\mathcal{B}(K)$ be a map of which the range contains all operators of rank $\leq 2$. Then the following statements are equivalent.

(1) $\Phi$ satisfies that $W([\Phi(A), \Phi(B)])=W([A, B])$ for any $A, B \in \mathcal{B}(H)$.

(2) $\operatorname{dim} H=\operatorname{dim} K$, and there exist $\varepsilon \in\{1,-1\}$, a functional $h: \mathcal{B}(H) \rightarrow \mathbb{C}$, a unitary operator $U \in \mathcal{B}(H, K)$, and a set $\mathcal{S}$ of operators in $\mathcal{B}(H)$, which consists of operators of the form $a P+b I$ for an orthogonal projection $P$ on $H$ if $\operatorname{dim} H \geq 3$, such that either

$$
\Phi(A)= \begin{cases}\varepsilon U A U^{*}+h(A) I \quad \text { if } A \in \mathcal{B}(H) \backslash \mathcal{S}, \\ -\varepsilon U A U^{*}+h(A) I \quad \text { if } A \in \mathcal{S},\end{cases}
$$

or

$$
\Phi(A)= \begin{cases}i \varepsilon U A^{t} U^{*}+h(A) I & \text { if } A \in \mathcal{B}(H) \backslash \mathcal{S}, \\ -i \varepsilon U A^{t} U^{*}+h(A) I & \text { if } A \in \mathcal{S},\end{cases}
$$

where $A^{t}$ is the transpose of $A$ with respect to an orthonormal basis of $H$.

An interesting open question is how to characterize non-linear maps on self-adjoint operators preserving numerical radius or numerical range of Lie product. In this paper, we solve this question for the case when the underline space $H$ is separable.

Let $H$ be a complex separable Hilbert space and $\Phi: \mathcal{B}_{s}(H) \rightarrow \mathcal{B}_{s}(H)$ a surjective map. Assume further that $\operatorname{dim} H \geq 3$. We show that:

(a) $\Phi$ satisfies $w(A B-B A)=w(\Phi(A) \Phi(B)-\Phi(B) \Phi(A))$ for any $A, B \in \mathcal{B}_{s}(H)$ if and only if there exist a unitary operator $U$ on $H$, a sign function $h: \mathcal{B}_{s}(H) \rightarrow\{1,-1\}$ and a functional $f: \mathcal{B}_{s}(H) \rightarrow \mathbb{R}$ such that $\Phi(T)=h(T) U T U^{*}+f(T) I$ for all $T \in \mathcal{B}_{s}(H)$ or $\Phi(T)=h(T) U T^{t} U^{*}+f(T) I$ for all $T \in \mathcal{B}_{s}(H)$ (See Theorem 2.1); 
(b) $\Phi$ satisfies $W(A B-B A)=W(\Phi(A) \Phi(B)-\Phi(B) \Phi(A))$ for all $A, B \in \mathcal{B}_{s}(H)$ if and only if there exist a unitary operator $U$ on $H$, a scalar $\varepsilon \in\{1,-1\}$, a subset $\mathcal{S} \subseteq \mathcal{D}(H)$, and a functional $f: \mathcal{B}_{s}(H) \rightarrow \mathbb{R}$ such that $\Phi(A)=\varepsilon U A U^{*}+f(A) I$ if $A \in \mathcal{B}_{s}(H) \backslash \mathcal{S}$, $\Phi(A)=-\varepsilon U A U^{*}+f(A) I$ if $A \in \mathcal{S}$, where $\mathcal{D}(H)$ is the set of all real linear combinations of a projection and the identity $I$ on $H$ (See Theorem 3.1).

When $\operatorname{dim} H=2$, not like to the maps on $\mathcal{B}(H)$ (See Theorem HLQ list above), the maps $\Phi: \mathcal{B}_{s}(H) \rightarrow \mathcal{B}_{s}(H)$ which preserve numerical range (radius) of Lie product may have some other forms. Note that, in the case $\operatorname{dim} H=2$ we have $\mathcal{D}(H)=\mathcal{B}_{2}(H)$. Identifying $\mathcal{B}_{s}(H)$ with the space $\mathbf{H}_{2}$ of all $2 \times 2$ Hermitian matrices, and define $\Psi$ on $\mathbf{H}_{2}$ by

$$
\left(\begin{array}{cc}
a & c+i d \\
c-i d & b
\end{array}\right) \mapsto\left(\begin{array}{cc}
a & -c+i d \\
-c-i d & b
\end{array}\right)
$$

It is easily checked that $\Psi$ preserves both the numerical range and the numerical radius of Lie product. However, no more other kind of maps can be added in as revealed by our result. In addition, the surjectivity assumption is not needed in the following result.

(c) A map $\Phi: \mathbf{H}_{2} \rightarrow \mathbf{H}_{2}$ preserves the numerical radius of Lie product if and only if it preserves the numerical range of Lie product, and in turn, if and only if there exist a unitary matrix $U \in M_{2}$, a sign function $h: \mathbf{H}_{2} \rightarrow\{1,-1\}$ and a functional $f: \mathcal{B}_{s}(H) \rightarrow \mathbb{R}$ such that either $\Phi(A)=h(A) U A^{\dagger} U^{*}+f(A) I$ for all $A \in \mathbf{H}_{2}$; or $\Phi(A)=h(A) U \Psi(A)^{\dagger} U^{*}+f(A) I$ for all $A \in \mathbf{H}_{2}$, where $(\cdot)^{\dagger}$ is one of the identity map and the transpose map (See Theorem 4.1).

The paper is organized as follows. We characterize the maps preserve the numerical radius of Lie product for the case $\operatorname{dim} H \geq 3$ in Section 2 and the maps preserving numerical range of Lie product for the case $\operatorname{dim} H \geq 3$ in Section 3. The last section is devoted to the case when $\operatorname{dim} H=2$.

\section{Preservers for numerical Radius of Lie product}

In the section, we devote to characterizing surjective maps on self-adjoint operators preserving numerical radius of Lie product for the case $\operatorname{dim} H \geq 3$. The following is the main result.

Theorem 2.1. Let $H$ be a separable complex Hilbert space of dimension at least three. A surjective map $\Phi: \mathcal{B}_{s}(H) \rightarrow \mathcal{B}_{s}(H)$ satisfies

$$
w(A B-B A)=w(\Phi(A) \Phi(B)-\Phi(B) \Phi(A))
$$

for all $A, B \in \mathcal{B}_{s}(H)$ if and only if there exist a unitary operator $U$ on $H$, a sign function $h: \mathcal{B}_{s}(H) \rightarrow\{1,-1\}$ and a functional $f: \mathcal{B}_{s}(H) \rightarrow \mathbb{R}$ such that either

$$
\Phi(T)=h(T) U T U^{*}+f(T) I
$$

for all $T \in \mathcal{B}_{s}(H)$; or

$$
\Phi(T)=h(T) U T^{t} U^{*}+f(T) I
$$


for all $T \in \mathcal{B}_{s}(H)$. Here $T^{t}$ is the transpose of $T$ with respect to an arbitrarily given orthonormal basis of $H$.

Before starting the proof of Theorem 2.1, we need a lemma.

Lemma 2.2. Let $H$ be a complex Hilbert space of dimension $\geq 2$ and $A, B$ be self-adjoint operators acting on $H$. Then the following statements are equivalent.

(1) $w(A C-C A)=w(B C-C B)$ for every $C \in \mathcal{B}_{s}(H)$.

(2) $w(A P-P A)=w(B P-P B)$ for every rank-1 projection $P$.

(3) $A+B$ or $A-B$ is a scalar.

Proof. $(3) \Rightarrow(1) \Rightarrow(2)$ are obvious. Let us check $(2) \Rightarrow(3)$.

Assume (2). For any rank-1 projection $P=x \otimes x$, write $A x=\alpha x+\beta y$, where normalized $y$ is orthogonal to $x$. Since $A$ is self-adjoint we have $\alpha=\langle A x, x\rangle \in \mathbb{R}$. Moreover, by selfadjointness of $A, A x \otimes x-x \otimes x A=A x \otimes x-x \otimes(A x)$. So relative to decomposition $H=[x, y] \oplus H_{1}$, the rank-2 operator $A x \otimes x-x \otimes x A$ is represented by a matrix

$$
\left(\begin{array}{cc}
0 & -\bar{\beta} \\
\beta & 0
\end{array}\right) \oplus 0,
$$

and hence $W(A x \otimes x-x \otimes x A)=i[-|\beta|,|\beta|]$ and $w(A x \otimes x-x \otimes x A)=|\beta|$. Decomposing likewise $B x=\alpha^{\prime} x+\beta^{\prime} z$ we obtain $W(B x \otimes x-x \otimes x B)=i\left[-\left|\beta^{\prime}\right|,\left|\beta^{\prime}\right|\right]$ and the numerical radius of $[B, x \otimes x]$ is $\left|\beta^{\prime}\right|$. Hence by $(2)$ we obtain $|\beta|=\left|\beta^{\prime}\right|$.

Since $A$ is self-adjoint and $\alpha=\langle A x, x\rangle \in \mathbb{R}$, it follows that $|\beta|^{2}=\|(A x-\langle A x, x\rangle x)\|^{2}=$ $\langle(A x-\langle A x, x\rangle x),(A x-\langle A x, x\rangle x)\rangle=\left\langle A^{2} x, x\right\rangle-\langle A x, x\rangle^{2}$. Similarly, for $B$ we obtain $\left|\beta^{\prime}\right|^{2}=$ $\left\langle B^{2} x, x\right\rangle-\langle B x, x\rangle^{2}$. If follows from $|\beta|^{2}=\left|\beta^{\prime}\right|^{2}$ that

$$
\left\langle A^{2} x, x\right\rangle-\left\langle B^{2} x, x\right\rangle=\langle A x, x\rangle^{2}-\langle B x, x\rangle^{2}
$$

for every normalized vector $x$. Let $y, z$ be two orthogonal normalized vectors. Then $x=$ $\frac{\sqrt{2}}{2}\left(e^{i \xi} y+z\right)$ is also normalized for every $\xi \in[-\pi, \pi]$. After inserting $x$ in Eq.(2.1) we obtain

$$
\begin{aligned}
0= & 2\left\langle A^{2}\left(e^{i \xi} y+z\right), e^{i \xi} y+z\right\rangle-2\left\langle B^{2}\left(e^{i \xi} y+z\right), e^{i \xi} y+z\right\rangle \\
& -\left(\left\langle A\left(e^{i \xi} y+z\right), e^{i \xi} y+z\right\rangle\right)^{2}+\left(\left\langle B\left(e^{i \xi} y+z\right), e^{i \xi} y+z\right\rangle\right)^{2} .
\end{aligned}
$$

Taking only the coefficient at $e^{2 i \xi}$ in the expansion of Eq.(2.2) in a Fourier series, Eq.(2.2) reduces into

$$
\langle B y, z\rangle^{2}=\langle A y, z\rangle^{2}
$$

for every pair of orthonormal $y, z$. So, for any $x \in H$ and $f \in[A x, x]^{\perp}$, we have $\langle B x, f\rangle=0$. This entails that $B x \in[A x, x]$. Thus, for any $x \in H$, there exist $\alpha_{x}, \beta_{x} \in \mathbb{C}$ such that $B x=$ $\alpha_{x} A x+\beta_{x} x$. By Eq.(2.3), we have $\langle A x, f\rangle^{2}=\left\langle\alpha_{x} A x, f\right\rangle^{2}=\alpha_{x}^{2}\langle A x, f\rangle^{2}$ holds for all $f \in[x]^{\perp}$, which implies that $\alpha_{x}= \pm 1$. It follows from $\left|\beta_{x}\right|\|x\| \leq\|B x\|+\left\|\alpha_{x} A x\right\| \leq(\|B\|+\|A\|)\|x\|$ that $\left|\beta_{x}\right| \leq\|B\|+\|A\|$. Therefore, $B$ is a regular local linear combination of $A$ and $I$, and then, by [14, $B$ is a linear combination of $A$ and $I$. So $B=\alpha A+\beta I$ with $\alpha \in\{-1,1\}$ and $\beta \in \mathbb{R}$, as desired. 
Proof of Theorem 2.1. The "if" part is obvious, we check the "only if" part.

Assume first that $\Phi$ is injective; then, $\Phi$ is bijective. Clearly $\Phi$ preserves zeros of Lie product. So, by [20], there exists a unitary or conjugate unitary operator $U$ such that, for any rank-1 positive operator $P=x \otimes x$ with unit vector $x \in H$, we have

$$
\Phi(P)=U\left(\lambda_{P} P+\mu_{P} I\right) U^{*}
$$

for some $\lambda_{P}, \mu_{P} \in \mathbb{R}$. Without loss of generality we can assume in the sequel that $U=I$. Taking any unit vectors $x, y$, which are orthogonal to each other, and let $Q=y \otimes y$ and $Z=(x+y) \otimes(x+y)$. It easily follows that in the orthogonal decomposition $H=[x, y] \oplus H_{1}$, where $[x, y]$ stands for the subspace spanned by $\{x, y\}$, we have $P Z-Z P=\left(\begin{array}{cc}0 & 1 \\ -1 & 0\end{array}\right) \oplus 0$, whose numerical range is $[-i, i]$ and so numerical radius is 1 . The same conclusion holds for the numerical range of $Q Z-Z Q$. Comparing the numerical radius of $P Z-Z P$ and $\Phi(P) \Phi(Z)-\Phi(Z) \Phi(P)$, we obtain

$$
1=w(\Phi(P) \Phi(Z)-\Phi(Z) \Phi(P))=\left|\lambda_{P} \lambda_{Z}\right| w(P Z-Z P)=\left|\lambda_{P} \lambda_{Z}\right| .
$$

This is possible only if $\lambda_{P} \lambda_{Z}= \pm 1$ since $\lambda_{P}, \lambda_{Z} \in \mathbb{R}$. Similarly we have $\lambda_{Q} \lambda_{Z}= \pm 1$. Hence $\lambda_{P}= \pm \lambda_{Q}$ for orthogonal $P, Q$. Now, given any rank-one self-adjoint operator $R$, there exists a rank-one self-adjoint operator $T$ which is orthogonal to $R$ and $P$. Similar to the above discussion, we have $\lambda_{T}= \pm \lambda_{R}$ and $\lambda_{T}= \pm \lambda_{P}$, so $\lambda_{P}= \pm \lambda_{R}$ for any $P, R$. It follows that $\lambda_{P}= \pm 1$.

Now, for arbitrary self-adjoint $A$,

$$
w(A x \otimes x-x \otimes x A)=\left|\lambda_{P}\right| w(\Phi(A) x \otimes x-x \otimes x \Phi(A))=w(\Phi(A) x \otimes x-x \otimes x \Phi(A))
$$

holds for every rank-1 projection $P=x \otimes x$. By Lemma 2.2, $\Phi(A)=\lambda_{A} A+\delta_{A} I$ for some scalar $\lambda_{A} \in\{-1,1\}$ and some scalar $\delta_{A}$.

Finally we show that one only needs the surjective assumption. Here we borrow an idea from [19]. If $\Phi(A)=\Phi(B)$, then

$$
\begin{aligned}
w(A C-C A) & =w(\Phi(A) \Phi(C)-\Phi(C) \Phi(A)) \\
& =w(\Phi(B) \Phi(C)-\Phi(C) \Phi(B))=w(B C-C B)
\end{aligned}
$$

for all $C \in \mathcal{B}_{s}(H)$. By Lemma 2.2 we get $B=\alpha A+\beta I$ for some $\alpha \in\{-1,1\}$ and $\beta \in \mathbb{R}$. On the other hand, for any $A$, there is some $D$ such that $\Phi(D)=-\Phi(A)$, which gives $w(D C-C D)=w(\Phi(D) \Phi(C)-\Phi(C) \Phi(D))=w(\Phi(A) \Phi(C)-\Phi(C) \Phi(A))=w(A C-C A)$ for all $C$. Again by Lemma 2.2, we get $D=\lambda A+\gamma I$ for some $\lambda \in\{-1,1\}$ and $\gamma \in \mathbb{R}$. For any $A, B \in \mathcal{B}_{s}(H)$, we say $A \sim B$ if $w(A C-C A)=w(B C-C B)$ for all $C \in \mathcal{B}_{s}(H)$. By Lemma 2.2, $\sim$ is an equivalent relation and $A \sim B$ if and only if $B=\alpha A+\beta I$ for some $\alpha \in\{-1,1\}$ and $\beta \in \mathbb{R}$. Let $\mathcal{E}_{A}=\left\{B \in \mathcal{B}_{s}(H): B \sim A\right\}$. For each equivalent class $\mathcal{E}_{A}$ pick a representative, for example $A$, and write $\mathcal{A}$ the set of these representatives. Since $\Phi$ is surjective, for each $A \in \mathcal{A}, \mathcal{E}_{A}$ and $\Phi^{-1}\left(\mathcal{E}_{A}\right)$ have the same cardinality $c$. Thus there exists a 
map $\Psi: \mathcal{B}_{s}(H) \rightarrow \mathcal{B}_{s}(H)$ which maps bijectively $\Phi^{-1}\left(\mathcal{E}_{A}\right)$ onto $\mathcal{E}_{A}$ for each $A \in \mathcal{A}$. Obviously, $\Psi$ is bijective and $\Psi(A) \sim \Phi(A)$ for all $A \in \mathcal{B}_{s}(H)$. Then

$$
w(\Psi(A) \Psi(B)-\Psi(B) \Psi(A))=w(\Phi(A) \Phi(B)-\Phi(B) \Phi(A))=w(A B-B A)
$$

for all $A, B \in \mathcal{B}_{s}(H)$. By the previous part of our proof of the theorem under the bijective assumption, $\Psi$ has the desired form, and hence $\Phi$ has the desired form as $\Phi(A) \sim \Psi(A)$. So Theorem 2.1 holds true, completing the proof.

\section{Preservers for numerical RANGe of Lie Product}

This section is devoted to characterizing maps that preserve the numerical range of Lie product of self-adjoint operators. Our main result is Theorem 3.1, which is not a direct corollary of Theorem 2.1 for numerical radius preservers, since much more effort should be paid to determine the structure of the sign function $h: \mathcal{B}_{s}(H) \rightarrow\{1,-1\}$.

Denote $\mathcal{D}$ the set of all real linear combinations of a projection and the identity $I$, that is, $\mathcal{D}=\left\{\alpha P+\delta I: P\right.$ is a projection in $\left.\mathcal{B}_{s}(H), \alpha, \delta \in \mathbb{R}\right\} \subset \mathcal{B}_{s}(H)$. It is clear that $\mathcal{D}$ is those self-adjoint operators that are also quadric algebraical operators.

Theorem 3.1. Let $H$ be a complex separable Hilbert space of dimension at least 3. A surjection $\Phi: \mathcal{B}_{s}(H) \rightarrow \mathcal{B}_{s}(H)$ satisfies

$$
W(A B-B A)=W(\Phi(A) \Phi(B)-\Phi(B) \Phi(A))
$$

for all $A, B \in \mathcal{B}_{s}(H)$ if and only if there exist a unitary operator $U$ on $H$, a scalar $\varepsilon \in\{1,-1\}$, a set $\mathcal{S} \subseteq \mathcal{D}$, and a functional $f: \mathcal{B}_{s}(H) \rightarrow \mathbb{R}$ such that

$$
\Phi(A)=\left\{\begin{array}{cll}
\varepsilon U A U^{*}+f(A) I & \text { if } & A \in \mathcal{B}_{s}(H) \backslash \mathcal{S} \\
-\varepsilon U A U^{*}+f(A) I & \text { if } & A \in \mathcal{S}
\end{array}\right.
$$

To prove the above result we need a lemma, which gives a characterization of the quadric algebraic self-adjoint operators, that is, the operators in $\mathcal{D}$, inn terms of the numerical range of Lie product.

Lemma 3.2. Let $H$ be a complex Hilbert space with $\operatorname{dim} H \geq 3$ and $A \in \mathcal{B}_{s}(H)$. Then the following statements are equivalent.

(1) $A \in \mathcal{D}$.

(2) $W(A B-B A)=-W(A B-B A)$ for all $B \in \mathcal{B}_{s}(H)$.

(3) $W(A B-B A)=-W(A B-B A)$ for all $B \in \mathcal{B}_{s}(H)$ of rank $\leq 2$.

Proof. (1) $\Rightarrow(2)$. Assume $A \in \mathcal{D}$, then $A=\alpha P+\gamma I$ for some projection $P$ and some scalars $\alpha, \gamma \in \mathbb{R}$. As the case $A=\alpha I$ is obvious, we may assume that, there exists a space decomposition $H=H_{1} \oplus H_{2}$ such that $A=\left(\begin{array}{cc}\alpha I_{H_{1}} & 0 \\ 0 & \beta I_{H_{2}}\end{array}\right)$ with $\operatorname{dim} H_{i}>0, i=1,2$, and $\alpha \neq \beta$. For any $B=\left(\begin{array}{ll}B_{11} & B_{12} \\ B_{12}^{*} & B_{22}\end{array}\right) \in \mathcal{B}_{s}\left(H_{1} \oplus H_{2}\right), A B-B A=(\alpha-\beta)\left(\begin{array}{cc}0 & B_{12} \\ -B_{12}^{*} & 0\end{array}\right)$. 
Let $U=\left(\begin{array}{cc}I_{H_{1}} & 0 \\ 0 & -I_{H_{2}}\end{array}\right)$; then $U$ is unitary and $U(A B-B A) U^{*}=-(A B-B A)$. So, we always have $W(A B-B A)=-W(A B-B A)$, that is, (2) is true.

$(2) \Rightarrow(3)$ is obvious.

$(3) \Rightarrow(1)$. Note that $A \in \mathcal{B}_{s}(H) \backslash \mathcal{D}$ if and only if the spectrum $\sigma(A)$ has at least three points, and in turn, if and only if there exists a vector $x$ such that $\left\{x, A x, A^{2} x\right\}$ is linearly independent. For such $x$, take an orthonormal basis $\left\{e_{1}, e_{2}, e_{3}\right\}$ of $\left[x, A x, A^{2} x\right]$ with $e_{1} \in[x]$ and $e_{2} \in[x, A x]$. Then, with respect to the space decomposition $H=\left[e_{1}\right] \oplus\left[e_{2}\right] \oplus\left[e_{3}\right] \oplus\left\{e_{1}, e_{2}, e_{3}\right\}^{\perp}, A$ has the matrix representation of the form

$$
A=\left(\begin{array}{cccc}
a_{11} & a_{21} & 0 & 0 \\
a_{21} & a_{22} & a_{32} & 0 \\
0 & a_{32} & a_{33} & A_{34} \\
0 & 0 & A_{34}^{*} & A_{44}
\end{array}\right)
$$

with $a_{11}, a_{22}, a_{33}$ real numbers, $a_{21}>0, a_{32}>0$ and $A_{44}=A_{44}^{*}$. Let

$$
B=\left(\begin{array}{cccc}
1 & \beta & 0 & 0 \\
\bar{\beta} & 0 & 0 & 0 \\
0 & 0 & 0 & 0 \\
0 & 0 & 0 & 0
\end{array}\right)
$$

with $\operatorname{Im} \beta=\frac{1}{2 i}(\beta-\bar{\beta}) \neq 0$. Then, $B$ is of rank two and

$$
A B-B A=\left(\begin{array}{cccc}
-2(\operatorname{Im} \beta) a_{21} & -a_{21}+\beta\left(a_{11}-a_{22}\right) & -\beta a_{32} & 0 \\
a_{21}-\bar{\beta}\left(a_{11}-a_{22}\right) & 2(\operatorname{Im} \beta) a_{21} & 0 & 0 \\
\bar{\beta} a_{32} & 0 & 0 & 0 \\
0 & 0 & 0 & 0
\end{array}\right)
$$

which is a rank-3 skew self-adjoint operator with zero trace. Clearly, $W(A B-B A) \neq$ $-W(A B-B A)$. Hence (3) implies (1).

\section{Proof of Theorem 3.1.}

Assume $\operatorname{dim} H \geq 3$. Then $\Phi$ satisfies the assumption of Theorem 2.1, and hence there exist a unitary operator or conjugate unitary operator $U$ on $H$, a sign function $h: \mathcal{B}_{s}(H) \rightarrow\{1,-1\}$ and a functional $f: \mathcal{B}_{s}(H) \rightarrow \mathbb{R}$ such that $\Phi(T)=h(T) U T U^{*}+f(T) I$ for all $T \in \mathcal{B}_{s}(H)$.

We assert that the case $U$ is a conjugate unitary operator cannot occur. Assume on the contrary that $\Phi(T)=h(T) U T U^{*}+f(T) I$ for any $T \in \mathcal{B}_{s}(H)$, where $U$ is conjugate. Take arbitrarily an orthonormal basis of $H$, one sees that there exists a unitary operator $V$ such that $\Phi(T)=h(T) V T^{t} V^{*}+f(T) I$ for any $T \in \mathcal{B}_{s}(H)$, where $T^{t}$ is the transpose of $T$ with 
respect to the given basis. Thus we have

$$
\begin{aligned}
W(A B-B A) & =W(\Phi(A) \Phi(B)-\Phi(B) \Phi(A)) \\
& =h(A) h(B) W\left(V A^{t} B^{t} V^{*}-V B^{t} A^{t} V^{*}\right) \\
& =h(A) h(B) W\left((B A-A B)^{t}\right) \\
& =-h(A) h(B) W(A B-B A) .
\end{aligned}
$$

Let $\{x, y, z\}$ be an orthonormal set of $H$ and consider the space decomposition $H=$ $[x, y, z] \oplus[x, y, z]^{\perp}$. For any scalars $\alpha, \beta, \gamma$ with $\alpha \beta \bar{\gamma}-\bar{\alpha} \bar{\beta} \gamma \neq 0$, and any real numbers $b_{11}, b_{22}, b_{33}$, let

$$
B=\left(\begin{array}{ccc}
b_{11} & \alpha & \gamma \\
\bar{\alpha} & b_{22} & \beta \\
\bar{\gamma} & \bar{\beta} & b_{33}
\end{array}\right) \oplus 0 \in \mathcal{B}_{s}(H)
$$

Then for any self-adjoint operator of the form

$$
A=\left(\begin{array}{ccc}
a_{1} & 0 & 0 \\
0 & a_{2} & 0 \\
0 & 0 & a_{3}
\end{array}\right) \oplus A_{2}
$$

with distinct $a_{1}, a_{2}, a_{3}$, we have $A B-B A=C_{1} \oplus 0$, where

$$
C_{1}=\left(\begin{array}{ccc}
0 & \left(a_{1}-a_{2}\right) \alpha & \left(a_{1}-a_{3}\right) \gamma \\
\left(a_{2}-a_{1}\right) \bar{\alpha} & 0 & \left(a_{2}-a_{3}\right) \beta \\
\left(a_{3}-a_{1}\right) \bar{\gamma} & \left(a_{3}-a_{2}\right) \bar{\beta} & 0
\end{array}\right) .
$$

As $\operatorname{det}\left(C_{1}\right)=\left(a_{1}-a_{2}\right)\left(a_{2}-a_{3}\right)\left(a_{3}-a_{1}\right)(\alpha \beta \bar{\gamma}-\bar{\alpha} \bar{\beta} \gamma) \neq 0, \sigma\left(C_{1}\right)=\left\{i t_{1}, i t_{2}, i t_{3}\right\}$ with $t_{i} \neq 0$, $i=1,2,3, t_{1} \leq t_{2} \leq t_{3}$ and $t_{1}+t_{2}+t_{3}=0$. So $W(A B-B A)=i\left[t_{1}, t_{3}\right]$ and $t_{1} \neq-t_{3}$. By Eq.(3.1) we obtain that

$$
-h(A) h(B)\left[i t_{1}, i t_{3}\right]=\left[i t_{1}, i t_{3}\right]
$$

and this forces $-h(A) h(B)=1$. If $h(B)=-1$, then $h(A)=1$ for all $A$ of the form in Eq.(3.3) and $h(B)=-1$ for all $B$ of the form in Eq.(3.2). Consequently, $h(B) h\left(B^{\prime}\right)=1$ for any $B, B^{\prime}$ of the form in Eq.(3.2).

Now take self-adjoint operators of rank two

$$
B=\left(\begin{array}{ccc}
0 & i & 1 \\
-i & 0 & 2 \\
1 & 2 & 0
\end{array}\right) \oplus 0 \text { and } B^{\prime}=\left(\begin{array}{ccc}
0 & 1+i & 1 \\
1-i & 0 & 2 i \\
1 & -2 i & 0
\end{array}\right) \oplus 0
$$

It is clear that $i\left(B B^{\prime}-B^{\prime} B\right)$ is a rank-3 self-adjoint operator and hence $W\left(B B^{\prime}-B^{\prime} B\right) \neq$ $-W\left(B B^{\prime}-B^{\prime} B\right)=-h(B) h\left(B^{\prime}\right) W\left(B B^{\prime}-B^{\prime} B\right)$, contradicting to Eq.(3.1).

So,

$$
\Phi(A)=h(A) U A U^{*}+f(A) I
$$

for all $A \in \mathcal{B}_{s}(H)$. 
It is clear by Lemma 3.2 that $h(A)$ can take any value of -1 and 1 if $A \in \mathcal{D}$. So, to complete the proof, we have to show that $h: \mathcal{B}_{s}(H) \rightarrow\{-1,1\}$ is constant on $\mathcal{B}_{s}(H) \backslash \mathcal{D}$.

By Lemma 3.2, for any $A \in \mathcal{B}_{s}(H) \backslash \mathcal{D}$, there exists rank-2 $B \in \mathcal{B}_{s}(H) \backslash \mathcal{D}$ such that $W(A B-B A) \neq-W(A B-B A)$. So we need only to show that $h(A)=h(B)$ holds for any rank-two $A, B \in \mathcal{B}_{s}(H) \backslash \mathcal{D}$.

Claim 1. For any orthonormal set $\{x, y, z\}$ and any nonzero real numbers $a, b, c, d, e, f$ with $a \neq b, c \neq d$ and $e \neq f$, we have $h(a x \otimes x+b y \otimes y)=h(c x \otimes x+d z \otimes z)=h(e y \otimes y+f z \otimes z)$.

Assume $A$ is a rank-2 self-adjoint not in $\mathcal{D}$. Then there exist orthonormal $x, y \in H$ and nonzero distinct real numbers $a, b$ such that $A=\left(\begin{array}{ll}a & 0 \\ 0 & b\end{array}\right) \oplus 0$ with respect to the space decomposition $H=[x, y] \oplus[x, y]^{\perp}$. Take arbitrarily two unit vectors $z, z^{\prime} \in[x, y]^{\perp}$ and nonzero complex numbers $\alpha, \beta, \gamma, \alpha^{\prime}, \beta^{\prime}, \gamma^{\prime}$ so that $\operatorname{Re}(\alpha \beta \bar{\gamma})=0$ and $\operatorname{Re}\left(\alpha^{\prime} \beta^{\prime} \bar{\gamma}^{\prime}\right)=0$, and let $B=B(x, y, z ; \alpha, \beta, \gamma)=\operatorname{Re}(x \otimes(\alpha y+\gamma z)+\beta y \otimes z), B^{\prime}=B\left(x, y, z^{\prime} ; \alpha^{\prime}, \beta^{\prime}, \gamma^{\prime}\right)=\operatorname{Re}\left(x \otimes\left(\alpha^{\prime} y+\right.\right.$ $\left.\left.\gamma^{\prime} z^{\prime}\right)+\beta^{\prime} y \otimes z^{\prime}\right)$. Then $A$ has the form in Eq.(3.1) and $B, B^{\prime}$ have the form in Eq.(3.2). By what proved previously, we see that both $B, B^{\prime}$ are of rank-2 and $h(B)=h(A)=h\left(B^{\prime}\right)$ as $W(A B-B A) \neq-W(A B-B A)$ and $W\left(A B^{\prime}-B^{\prime} A\right) \neq-W\left(A B^{\prime}-B^{\prime} A\right)$. It is also clear that

$$
h(a x \otimes x+b y \otimes y)=h(B(x, y, z ; \alpha, \beta, \gamma))=h\left(B\left(\pi(x, y, z) ; \alpha_{1}, \beta_{1}, \gamma_{1}\right)\right)
$$

holds for any permutation $\pi(x, y, z)$ of $(x, y, z)$ and any nonzero numbers $\alpha_{1}, \beta_{1}, \gamma_{1}$ with $\operatorname{Re} \alpha_{1} \beta_{1} \bar{\gamma}_{1}=0$. For example,

$$
h(a x \otimes x+b y \otimes y)=h(B(x, y, z ; \alpha, \beta, \gamma))=h\left(B\left(z, x, y ; \alpha_{1}, \beta_{1}, \gamma_{1}\right)\right) .
$$

It follows that

$$
h(a x \otimes x+b y \otimes y)=h(c x \otimes x+d z \otimes z)=h(e y \otimes y+f z \otimes z)
$$

hold for any orthonormal set $\{x, y, z\}$ and any nonzero real numbers $a, b, c, d, e, f$ with $a \neq$ $b, c \neq d$ and $e \neq f$. So Claim 1 is true.

Claim 2. If $\operatorname{dim} H \geq 4$, then $h(A)=h(B)$ holds for any rank-2 $A, B \in \mathcal{B}_{s}(H) \backslash \mathcal{D}$.

Let $A=a x \otimes x+b y \otimes y$ and $B=c u \otimes u+d v \otimes v$ be any two rank-2 self-adjoint operators that are not in $\mathcal{D}$, where $x \perp y$ and $u \perp v$. $\operatorname{dim} H \geq 4$ implies that $[x, y, u] \neq H$. Take $y^{\prime} \in[x, y, u]^{\perp}$. By Claim 1 we have

$$
\Phi(a x \otimes x+b y \otimes y)=\Phi\left(b y \otimes y+c y^{\prime} \otimes y^{\prime}\right) .
$$

So, replacing $y$ by $y^{\prime}$ if necessary, we may assume that $y \perp u$ in the sequel.

If $[x, y, u, v] \neq H$, one can pick a unit vector $z \in[x, y, u, v]^{\perp}$. Then, by Claim 1 or Eq.(3.4),

$$
\begin{aligned}
h(A) & =h(a x \otimes x+b y \otimes y)=h(a y \otimes y+b z \otimes z) \\
& =h(c u \otimes u+b z \otimes z)=h(c u \otimes u+d v \otimes v)=h(B) .
\end{aligned}
$$


If $[x, y, u, v]=H$, then $\operatorname{dim} H=4$. Take unit vectors $z \in[x, y, u]^{\perp}$ and $z^{\prime} \in[y, u, v]^{\perp}$. Applying Claim 1 again, we see that

$$
\begin{aligned}
h(A) & =h(a x \otimes x+b y \otimes y)=h(a y \otimes y+b z \otimes z) \\
& =h(a y \otimes y+b u \otimes u)=h\left(c u \otimes u+d z^{\prime} \otimes z^{\prime}\right) \\
& =h(c u \otimes u+d v \otimes v)=h(B) .
\end{aligned}
$$

Finally, let us consider the case $\operatorname{dim} H=3$.

Claim 3. If $\operatorname{dim} H=3$, then $h(A)=h(B)$ holds for any rank-2 $A, B \in \mathcal{B}_{s}(H) \backslash \mathcal{D}$.

Assume that $\operatorname{dim} H=3$ and write $A=a x \otimes x+b y \otimes y$ and $B=c u \otimes u+d v \otimes v$, where $x \perp y, u \perp v$. If $[x, y, u, v] \neq H$, then $[x, y]=[u, v]$. It is obvious $h(A)=h(B)$ whenever $u$ is linearly dependent to $x$ or $y$. So we may assume that $u, v \notin[x] \cup[y]$. Pick a unit vector $z \in[x, y]^{\perp}$. By Claim 1 we see that $h(A)=h(a x \otimes x+b y \otimes y)=h(a z \otimes z+b y \otimes y)$ and $h(B)=h(c u \times u+d v \otimes v)=h(c z \otimes z+d v \otimes v)$. It reduces to consider $A^{\prime}=a z \otimes z+b y \otimes y$, $B^{\prime}=c z \otimes z+d v \otimes v$. Note that $[z, y, v]=H$. So we may always require that $[x, y, u, v]=H$.

Take unit vector $z \in[x, y]^{\perp}$; then $A$ and $B$ have matrix representations

$$
A=\left(\begin{array}{lll}
a & 0 & 0 \\
0 & b & 0 \\
0 & 0 & 0
\end{array}\right) \quad \text { and } B=\left(\begin{array}{ccc}
\xi_{1} & \alpha & \gamma \\
\bar{\alpha} & \xi_{2} & \beta \\
\bar{\gamma} & \bar{\beta} & \xi_{3}
\end{array}\right)
$$

with $a, b, 0$ are distinct to each other, $B$ has three distinct eigvalues, $\left(\gamma, \beta, \xi_{3}\right) \neq(0,0,0)$. If $\alpha=\beta=\gamma=0$ or $\operatorname{Im}(\alpha \beta \bar{\gamma}) \neq 0$, clearly we already have $h(B)=h(A)$ (see the argument after Eqs. (3.3)-(3.4)).

In the sequel assume that $(\alpha, \beta, \gamma) \neq(0,0,0)$ but $\operatorname{Im}(\alpha \beta \bar{\gamma})=0$.

Subcase 1. Two of $\alpha, \beta, \gamma$ are 0 .

Without loss of generality, say $\beta=\gamma=0$. Then

$$
B=\left(\begin{array}{ccc}
\bar{\alpha} & \alpha & 0 \\
\bar{\alpha} & k \alpha & 0 \\
0 & 0 & \xi_{3}
\end{array}\right)
$$

for some $k \neq 0$ as $\operatorname{rank} B=2$ and $\xi_{3} \neq 0$. Let

$$
C_{t, s}=\left(\begin{array}{ccc}
0 & t & i \\
t & 0 & s \\
-i & s & 0
\end{array}\right)
$$

for nonzero $t, s \in \mathbb{R}$. By the previous discussion we have $h(A)=h\left(C_{t, s}\right)$. Consider

$$
B C_{t, s}-C_{t, s} B=\left(\begin{array}{ccc}
t(\alpha-\bar{\alpha}) & t\left(\frac{\bar{\alpha}}{k}-k \alpha\right) & \frac{i \bar{\alpha}}{k}+s \alpha-i \xi_{3} \\
-t\left(\frac{\bar{\alpha}}{k}-k \alpha\right) & -t(\alpha-\bar{\alpha}) & i \bar{\alpha}+s k \alpha-s \xi_{3} \\
\frac{i \bar{\alpha}}{k}-s \bar{\alpha}-i \xi_{3} & i \alpha-s k \alpha+s \xi_{3} & 0
\end{array}\right)
$$

It is clear that $\operatorname{det}\left(B C_{t, s}-C_{t, s} B\right) \neq 0$ for some $t, s$ whenever $\alpha \notin \mathbb{R}$ or $k \alpha \neq \frac{\bar{\alpha}}{k}$ or $k \alpha \neq \xi_{3}$ or $\xi_{3} \neq \frac{\bar{\alpha}}{k}$, and in this case we have $h(B)=h\left(C_{t, s}\right)=h(A)$. If $\alpha$ is real and $k \alpha=\frac{\bar{\alpha}}{k}=\xi_{3}$, then, 
up to a real scalar multiple, $B$ has the form

$$
B=\left(\begin{array}{lll}
1 & 1 & 0 \\
1 & 1 & 0 \\
0 & 0 & 1
\end{array}\right)
$$

Let

$$
C=\left(\begin{array}{ccc}
1 & 1 & 1+i \\
1 & 2 & 1-i \\
1-i & 1+i & 0
\end{array}\right)
$$

Then $\operatorname{Im}(1 \cdot(1-i) \overline{(1+i)})=-2 i \neq 0$ and hence $h(C)=h(A)$. Since $\operatorname{det}(B C-C B)=-4 i \neq 0$, we also have $h(B)=h(C)$. So, again we get $h(B)=h(A)$, as desired.

Subcase 2. One of $\alpha, \beta, \gamma$ is 0 .

Without loss of generality, say $\beta=0$. Then, as $\operatorname{rank} B=2, \operatorname{det} B=\xi_{1} \xi_{2} \xi_{3}-|\gamma|^{2} \xi_{2}-|\alpha|^{2} \xi_{3}=$ 0 . Thus there are scalars $c, d$ with $d \neq 0$ such that $c \xi_{1}=\bar{\gamma}-d \alpha, \xi_{2}=-\frac{c}{d} \alpha$ and $\xi_{3}=c \gamma$.

Clearly, $\xi_{2}=0 \Leftrightarrow \xi_{3}=0 \Leftrightarrow c=0$, and in this case we have

$$
B=\left(\begin{array}{ccc}
\xi_{1} & \alpha & \gamma \\
\bar{\alpha} & 0 & 0 \\
\bar{\gamma} & 0 & 0
\end{array}\right)
$$

Let

$$
C_{t, s, p}=\left(\begin{array}{ccc}
0 & t & i p \\
t & 0 & s \\
-i p & s & 0
\end{array}\right)
$$

for nonzero real numbers $t, s, p$; then $h(A)=h\left(C_{t, s, p}\right)$. Now

$$
B C_{t, s, p}-C_{t, s, p} B=\left(\begin{array}{ccc}
t(\alpha-\bar{\alpha})-i p(\gamma+\bar{\gamma}) & \xi_{1} t+\gamma s & i \xi_{1} p+\alpha s \\
-\xi_{1} t-\bar{\gamma} s & -t(\alpha-\bar{\alpha}) & i \bar{\alpha} p-\gamma t \\
i \xi_{1} p-\bar{\alpha} s & t \bar{\gamma}+i \alpha p & i p(\gamma+\bar{\gamma})
\end{array}\right)
$$

If $\xi_{1} \neq 0$ (in this case the coefficients of $s p^{2}$ and $t^{2} s$ of $\operatorname{det}\left(B C_{t, s, p}-C_{t, s, p} B\right)$ are nonzero), or if $\xi_{1}=0$ but one of $\alpha-\bar{\alpha}$ and $\gamma+\bar{\gamma}$ is nonzero (in this case the coefficient of $t^{3}$ or $p^{3}$ is nonzero), it is sure that $B C_{t, s, p}-C_{t, s, p} B$ is of rank three for some $t, s, p$ and hence $h(B)=h\left(C_{t, s, p}\right)=h(A)$. If

$$
B=\left(\begin{array}{ccc}
0 & \alpha & i \delta \\
\alpha & 0 & 0 \\
-i \delta & 0 & 0
\end{array}\right)
$$

for some nonzero real numbers $\alpha, \delta$, let

$$
D_{t, s, p}=\left(\begin{array}{ccc}
0 & i t & p \\
-i t & 0 & s \\
p & s & 0
\end{array}\right)
$$


for nonzero real numbers $t, s, p$. Then

$$
B D_{t, s, p}-D_{t, s, p} B=\left(\begin{array}{ccc}
2 i(\delta p-\alpha t) & i \delta s & \alpha s \\
i \delta s & 2 i \alpha t & \alpha p-\delta t \\
-\alpha s & \delta t-\alpha p & -2 i \delta p
\end{array}\right)
$$

which is of rank three for some suitable choice of $t, s, p$ as the coefficients of $t^{3}$ and $p^{3}$ of $\operatorname{det}\left(B D_{t, s, p}-D_{t, s, p} B\right)$ are nonzero. Therefore, we have $h(B)=h\left(D_{t, s, p}\right)=h(A)$.

Assume that $c \neq 0$; then $\xi_{1}=\frac{1}{c}(\bar{\gamma}-d \bar{\alpha}), \xi_{2}=-\frac{c}{d} \alpha, \xi_{3}=c \gamma$ are real,

$$
B=\left(\begin{array}{ccc}
\frac{1}{c}(\bar{\gamma}-d \bar{\alpha}) & \alpha & \gamma \\
\bar{\alpha} & -\frac{c}{d} \alpha & 0 \\
\bar{\gamma} & 0 & c \gamma
\end{array}\right)
$$

and, for $C_{t, s, p}$ in Eq.(3.5), we have

$$
\begin{aligned}
& B C_{t, s, p}-C_{t, s, p} B \\
= & \left(\begin{array}{ccc}
t(\alpha-\bar{\alpha})-i p(\gamma+\bar{\gamma}) & \left(\frac{\bar{\gamma}}{c}-\frac{d \bar{\alpha}}{c}+\frac{c \alpha}{d}\right) t+\gamma s & i\left(\frac{\bar{\gamma}}{c}-\frac{d \bar{\alpha}}{c}-c \gamma\right) p+\alpha s \\
-\left(\frac{\bar{\gamma}}{c}-\frac{d \bar{\alpha}}{c}+\frac{c \alpha}{d}\right) t-\bar{\gamma} s & -t(\alpha-\bar{\alpha}) & i \bar{\alpha} p-\gamma t-c\left(\frac{\alpha}{d}+\gamma\right) s \\
i\left(\frac{\bar{\gamma}}{c}-\frac{d \bar{\alpha}}{c}-c \gamma\right) p-\bar{\alpha} s & i \alpha p+t \bar{\gamma}+c\left(\frac{\alpha}{d}+\gamma\right) s & i p(\gamma+\bar{\gamma})
\end{array}\right) .
\end{aligned}
$$

Note that the coefficients of $t^{3}, s^{3}$ and $p^{3}$ in $\operatorname{det}\left(B C_{t, s, p}-C_{t, s, p} B\right)$ are respectively $|\gamma|^{2}(\alpha-$ $\bar{\alpha}), c\left(\frac{\alpha}{d}+\gamma\right)(\bar{\alpha} \gamma-\alpha \bar{\gamma})$ and $-i|\alpha|^{2}(\gamma+\bar{\gamma})$.

It is clear that, if $\alpha$ or $i \gamma$ are not real; or in the case that both $\alpha$ and $i \gamma$ are real, but $\xi_{2} \neq \xi_{3}$, then $B C_{t, s, p}-C_{t, s, p} B$ is rank-3 for suitable choice of real numbers $t, s, p$ and hence $h(B)=h\left(C_{t, s, p}\right)=h(A)$.

If $\alpha, i \gamma$ are real and $\xi_{2}=\xi_{3}$ but $\xi_{1} \neq \xi_{2}$, then

$$
=\left(\begin{array}{ccc}
B C_{t, s, p}-C_{t, s, p} B \\
0 & \left(\frac{\bar{\gamma}}{c}-\frac{d \bar{\alpha}}{c}+\frac{c \alpha}{d}\right) t+\gamma s & i\left(\frac{\bar{\gamma}}{c}-\frac{d \bar{\alpha}}{c}-c \gamma\right) p+\alpha s \\
-\left(\frac{\bar{\gamma}}{c}-\frac{d \bar{\alpha}}{c}+\frac{c \alpha}{d}\right) t-\bar{\gamma} s & 0 & i \bar{\alpha} p-\gamma t \\
i\left(\frac{\bar{\gamma}}{c}-\frac{d \bar{\alpha}}{c}-c \gamma\right) p-\bar{\alpha} s & i \alpha p+t \bar{\gamma} & 0
\end{array}\right) .
$$

As the coefficient of $t^{2} p$ in $\operatorname{det}\left(B C_{t, s, p}-C_{t, s, p} B\right)$ is $-i\left(\xi_{1}-\xi_{2}\right)^{2} \bar{\gamma} \neq 0$, we still have $h(B)=h(A)$.

If $\alpha, i \gamma$ are real and $\xi_{1}=\xi_{2}=\xi_{3}$, then $B$ has the form

$$
B=\left(\begin{array}{ccc} 
\pm \sqrt{\alpha^{2}+\delta^{2}} & \alpha & i \delta \\
\alpha & \pm \sqrt{\alpha^{2}+\delta^{2}} & 0 \\
-i \delta & 0 & \pm \sqrt{\alpha^{2}+\delta^{2}}
\end{array}\right)
$$

with nonzero $\alpha, \delta \in \mathbb{R}$. Then, for $D_{t, s, p}$ in Eq.(3.6), consider

$$
B D_{t, s, p}-D_{t, s, p} B=\left(\begin{array}{ccc}
2 i(\delta p-\alpha t) & i \delta s & \alpha s \\
i \delta s & 2 i \alpha t & \alpha p-\delta t \\
-\alpha s & \delta t-\alpha p & -2 i \delta p
\end{array}\right)
$$

for nonzero real numbers $t, s, p$. As the coefficient of $t^{3}$ in $\operatorname{det}\left(B D_{t, s, p}-D_{t, s, p} B\right)$ is $-2 i \alpha \delta^{2} \neq 0$, and hence one gets $h(B)=h(A)$ again. 
Subcase 3. All $\alpha, \beta, \gamma$ are nonzero.

Since $\operatorname{det}(B)=0$, there are scalars $c, d$ such that $\left(\bar{\gamma}, \bar{\beta}, \xi_{3}\right)=\left(c \xi_{1}+d \bar{\alpha}, c \alpha+d \xi_{2}, c \gamma+d \beta\right)$. It follows that

$$
\left\{\begin{array}{l}
\gamma=\bar{c} \xi_{1}+\bar{d} \alpha \\
\beta=\bar{c} \bar{\alpha}+\bar{d} \xi_{2} \\
\xi_{3}=|c|^{2} \xi_{1}+c \bar{d} \alpha+\bar{c} d \bar{\alpha}+|d|^{2} \xi_{2}
\end{array}\right.
$$

As $\alpha \beta \bar{\gamma} \in \mathbb{R}$, we get

$$
(c \bar{d} \alpha-\bar{c} d \bar{\alpha})\left(|\alpha|^{2}-\xi_{1} \xi_{2}\right)=0
$$

However, $|\alpha|^{2}-\xi_{1} \xi_{2}=0$ implies that $\xi_{1}=\frac{\bar{\alpha}}{k}, \xi_{2}=k \alpha$ for some scalar $k$, which entails that $\beta=k \gamma$ and hence $B$ is of rank-1, a contradiction. So $|\alpha|^{2}-\xi_{1} \xi_{2} \neq 0$ and then we must have $c \bar{d} \alpha-\bar{c} d \bar{\alpha}=0$. Discussing similarly, we get

$$
\left\{\begin{array}{l}
|\alpha|^{2}-\xi_{1} \xi_{2} \neq 0 \\
|\beta|^{2}-\xi_{2} \xi_{3} \neq 0 \\
|\gamma|^{2}-\xi_{1} \xi_{3} \neq 0
\end{array}\right.
$$

Let $C_{t, s, p}$ be as in Eq.(3.5). As

$$
\begin{aligned}
B C_{t, s, p}-C_{t, s, p} B \\
=\left(\begin{array}{ccc}
(\alpha-\bar{\alpha}) t-i(\gamma+\bar{\gamma}) p & \left(\xi_{1}-\xi_{2}\right) t+\gamma s-i \bar{\beta} p & i\left(\xi_{1}-\xi_{3}\right) p+\alpha s-\beta t \\
-\left(\xi_{1}-\xi_{2}\right) t-\bar{\gamma} s-i \beta p & -(\alpha-\bar{\alpha}) t+(\beta-\bar{\beta}) s & \left(\xi_{2}-\xi_{3}\right) s+i \bar{\alpha} p-\gamma t \\
i\left(\xi_{1}-\xi_{3}\right) p+\bar{\beta} t-\bar{\alpha} s & -\left(\xi_{2}-\xi_{3}\right) s+\bar{\gamma} t+i \alpha p & i(\gamma+\bar{\gamma}) p-(\beta-\bar{\beta}) s
\end{array}\right),
\end{aligned}
$$

we see that the coefficients of $t^{3}, s^{3}, p^{3}$ in $\operatorname{det}\left(B C_{t, s, p}-C_{t, s, p} B\right)$ are respectively

$$
\left\{\begin{array}{l}
c_{t}=\left(\xi_{1}-\xi_{2}\right)(\beta \bar{\gamma}-\bar{\beta} \gamma)+(\alpha-\bar{\alpha})\left(|\gamma|^{2}-|\beta|^{2}\right), \\
c_{s}=\left(\xi_{2}-\xi_{3}\right)(\alpha \bar{\gamma}-\bar{\alpha} \gamma)+(\beta-\bar{\beta})\left(|\alpha|^{2}-|\gamma|^{2}\right), \\
c_{p}=i\left(\xi_{1}-\xi_{3}\right)(\bar{\alpha} \bar{\beta}+\alpha \beta)+i(\bar{\gamma}+\gamma)\left(|\beta|^{2}-|\alpha|^{2}\right) .
\end{array}\right.
$$

If one of $c_{t}, c_{s}, c_{p}$ is nonzero, then $\operatorname{det}\left(B C_{t, s, p}-C_{t, s, p} B\right) \neq 0$ for some choice of $t, s, p$, which implies that $h(B)=h\left(C_{t, s, p}\right)=h(A)$. Assume

$$
c_{t}=c_{s}=c_{p}=0
$$

Considering the coefficients $d_{t}, d_{s}$ and $d_{p}$ of $t^{3}, s^{3}$ and $p^{3}$ in $\operatorname{det}\left(B D_{t, s, p}-D_{t, s, p} B\right)$ with $D_{t, s, p}$ as in Eq.(3.6) one gets

$$
\left\{\begin{array}{l}
d_{t}=i\left(\xi_{1}-\xi_{2}\right)(\beta \bar{\gamma}+\bar{\beta} \gamma)+i(\alpha+\bar{\alpha})\left(|\gamma|^{2}-|\beta|^{2}\right), \\
d_{s}=\left(\xi_{2}-\xi_{3}\right)(\alpha \bar{\gamma}-\bar{\alpha} \gamma)+(\beta-\bar{\beta})\left(|\alpha|^{2}-|\gamma|^{2}\right)=0 \\
d_{p}=\left(\xi_{1}-\xi_{3}\right)(\bar{\alpha} \bar{\beta}-\alpha \beta)+(\bar{\gamma}-\gamma)\left(|\beta|^{2}-|\alpha|^{2}\right) .
\end{array}\right.
$$

If one of $d_{t}, d_{p}$ is nonzero, then $h(B)=h(A)$. Assume that

$$
d_{t}=d_{s}=d_{p}=0
$$


Let

$$
E_{t, s, p}=\left(\begin{array}{ccc}
0 & t & p \\
t & 0 & i s \\
p & -i s & 0
\end{array}\right)
$$

for nonzero real numbers $t, s, p$. The coefficients $e_{t}, e_{s}$ and $e_{p}$ of $t^{3}, s^{3}$ and $p^{3}$ in $\operatorname{det}\left(B E_{t, s, p}-\right.$ $\left.E_{t, s, p} B\right)$ are

$$
\left\{\begin{array}{l}
e_{t}=\left(\xi_{1}-\xi_{2}\right)(\beta \bar{\gamma}-\bar{\beta} \gamma)+(\alpha-\bar{\alpha})\left(|\gamma|^{2}-|\beta|^{2}\right)=0, \\
e_{s}=i\left(\xi_{2}-\xi_{3}\right)(\alpha \bar{\gamma}+\bar{\alpha} \gamma)+i(\beta+\bar{\beta})\left(|\alpha|^{2}-|\gamma|^{2}\right), \\
e_{p}=\left(\xi_{1}-\xi_{3}\right)(\bar{\alpha} \bar{\beta}-\alpha \beta)+(\bar{\gamma}-\gamma)\left(|\beta|^{2}-|\alpha|^{2}\right)=0 .
\end{array}\right.
$$

If $e_{s} \neq 0$, then we get $h(B)=h(A)$. Assume

$$
e_{s}=0
$$

Then, by Eqs.(3.9)-(3.10), and Eq.(3.12), it is easily checked that

$$
\left\{\begin{array}{l}
\left(\xi_{1}-\xi_{2}\right) \beta \bar{\gamma}+\alpha\left(|\gamma|^{2}-|\beta|^{2}\right)=0 \\
\left(\xi_{2}-\xi_{3}\right) \alpha \bar{\gamma}+\beta\left(|\alpha|^{2}-|\gamma|^{2}\right)=0 \\
\left(\xi_{1}-\xi_{3}\right) \alpha \beta+\gamma\left(|\beta|^{2}-|\alpha|^{2}\right)=0
\end{array}\right.
$$

As $\alpha \beta \bar{\gamma}$ is real, we see from Eq.(3.13) that both $\alpha^{2}, \beta^{2}$ are real and hence $\alpha \in \mathbb{R}$ or $\alpha \in i \mathbb{R}$ $(\beta \in \mathbb{R}$ or $\beta \in i \mathbb{R})$. It follows that there are four cases may occur, that is,

$$
\begin{cases}1^{\circ} & \alpha, \beta, \gamma \in \mathbb{R} . \\ 2^{\circ} & \alpha, \beta \in i \mathbb{R}, \gamma \in \mathbb{R} . \\ 3^{\circ} & \beta, \gamma \in i \mathbb{R}, \alpha \in \mathbb{R} . \\ 4^{\circ} & \alpha, \gamma \in i \mathbb{R}, \beta \in \mathbb{R} .\end{cases}
$$

If $\xi_{1}=\xi_{2}=\xi_{3}=\xi$, then $|\alpha|=|\beta|=|\gamma|$ by Eq.(3.13). On the other hand, by Eq.(3.7), $\xi\left(1-|c|^{2}-|d|^{2}\right)=2 c \bar{d} \alpha$. Thus $\xi=0$ or $1-|c|^{2}-|d|^{2}=0$ implies that $c=0$ or $d=0$. Without loss of generality, say $c=0$; then $d \neq 0$ and $\xi=|d|^{2} \xi \neq 0$, which gives $d=e^{i \theta}$ and $|\xi|=|d \beta|=|\alpha|$, contradicting the fact that $|\alpha|^{2} \neq \xi_{1} \xi_{2}=\xi^{2}=|\xi|^{2}$ (see Eq.(3.8)).

So we have $\xi\left(1-|c|^{2}-|d|^{2}\right)=2 c \bar{d} \alpha \neq 0$,

$$
\xi=\frac{\gamma-\bar{d} \alpha}{c}=\frac{\beta-\bar{c} \bar{\alpha}}{\bar{d}}=\frac{2 c \bar{d} \alpha}{1-|c|^{2}-|d|^{2}}=\frac{2 \bar{c} d \bar{\alpha}}{1-|c|^{2}-|d|^{2}}
$$

and

$$
B=\left(\begin{array}{ccc}
\frac{2 c \bar{d} \alpha}{1-|c|^{2}-|d|^{2}} & \alpha & \left(2|c|^{2}+1\right) \bar{d} \alpha \\
\bar{\alpha} & \frac{2 c \bar{d} \alpha}{1-|c|^{2}-|d|^{2}} & \left(2|d|^{2}+1\right) \bar{c} \bar{\alpha} \\
\left(2|c|^{2}+1\right) d \bar{\alpha} & \left(2|d|^{2}+1\right) c \alpha & \frac{2 c \bar{d} \alpha}{1-|c|^{2}-|d|^{2}}
\end{array}\right) .
$$

It follows that $\left(2|c|^{2}+1\right)|d|=\left(2|d|^{2}+1\right)|c|=1$ as $|\alpha|=|\beta|=|\gamma|$. Thus $2|c|+\frac{1}{|c|}=2|d|+\frac{1}{|d|}=$ $\frac{1}{c c d \mid}$. Note that $|c|=\frac{1}{2|d|^{2}+1}$ and $|d|=\frac{1}{2|c|^{2}+1}$. So one gets $(|c|+|d|)(|c|-|d|)=|c|-|d|$, which gives further that either $|c|=|d|$ or $|c|+|d|=1$. If $|c| \neq|d|$, we must have $|c|+|d|=1$ and hence $0<1-|c|=|d|=\frac{1}{2|c|^{2}+1}$. Then we obtain $|c|\left(2|c|^{2}-2|c|+1\right)=0$. As we always have $2|c|^{2}-2|c|+1>0$, one sees that $c=0$, a contradiction. Therefore, we have $|c|=|d|=k$. 
Since $\left(2 k^{2}+1\right) k=1$, we see that $k \approx 0.5898$. Write $\alpha=|\alpha| e^{i \theta_{1}}, c=k e^{i \theta_{2}}$ and $d=k e^{i \theta_{3}}$. Now $c \bar{d} \alpha$ is real implies that $\theta_{1}+\theta_{2}-\theta_{3}$ is 0 or $\pi$. Replacing $B$ by $-B$ if necessary we may assume that $\theta_{1}+\theta_{2}-\theta_{3}=0$ and thus $d=k e^{i\left(\theta_{1}+\theta_{2}\right)}$. Without loss of generality, let $|\alpha|=1$. Notice that $\left(2 k^{2}+1\right) k=1$. Then $B$ becomes to

$$
B=\left(\begin{array}{ccc}
\frac{2 k^{2}}{1-2 k^{2}} & e^{i \theta_{1}} & e^{-i \theta_{2}} \\
e^{-i \theta_{1}} & \frac{2 k^{2}}{1-2 k^{2}} & e^{-i\left(\theta_{1}+\theta_{2}\right)} \\
e^{i \theta_{2}} & e^{i\left(\theta_{1}+\theta_{2}\right)} & \frac{2 k^{2}}{1-2 k^{2}}
\end{array}\right)
$$

with $\frac{2 k^{2}}{1-2 k^{2}} \approx 2.2868$. But then $0=\operatorname{det}(B)=\left(\frac{2 k^{2}}{1-2 k^{2}}\right)^{3}-3\left(\frac{2 k^{2}}{1-2 k^{2}}\right)+2 \approx 7.0983>0$, a contradiction. Therefore $\xi_{1}, \xi_{2}, \xi_{3}$ are not all the same. Keep this in mind below, we can show that $h(B)=h(A)$ holds.

For example, consider the Case $2^{\circ}$, that is, $\alpha \in \mathbb{R}, \beta, \gamma \in i \mathbb{R}$.

In this case, for $t, s, p \in \mathbb{C}$, let

$$
F_{t, s, p}=\left(\begin{array}{ccc}
0 & t & p \\
\bar{t} & 0 & s \\
\bar{p} & \bar{s} & 0
\end{array}\right) .
$$

Then

$$
\begin{aligned}
B F_{t, s, p}-F_{t, s, p} B & \left(\begin{array}{ccc}
\alpha(\bar{t}-t)+i \gamma(p+\bar{p}) & \left(\xi_{1}-\xi_{2}\right) t+i \gamma \bar{s}+i \beta p & \left(\xi_{1}-\xi_{3}\right) p-\alpha s-i \beta t \\
\left(\xi_{2}-\xi_{1}\right) \bar{t}+i \beta \bar{p}+i \gamma s & -\alpha(\bar{t}-t)+i \beta(s+\bar{s}) & \left(\xi_{2}-\xi_{3}\right) s+\alpha p-i \gamma \bar{t} \\
\left(\xi_{3}-\xi_{1}\right) \bar{p}-i \beta \bar{t}-\alpha \bar{s} & \left(\xi_{3}-\xi_{2}\right) \bar{s}-i \gamma t-\alpha \bar{p} & -i \gamma(p+\bar{p})-i \beta(s+\bar{s})
\end{array}\right) .
\end{aligned}
$$

Consider the term of $\operatorname{det}\left(\left[B, F_{t, s, p}\right]\right)$ that contains only $t$, which is

$$
\left(\left(\xi_{1}-\xi_{2}\right) \beta \gamma+\alpha\left(\beta^{2}-\gamma^{2}\right)\right)\left(t^{2} \bar{t}-\bar{t}^{2} t\right)=2\left(\xi_{1}-\xi_{2}\right) \beta \gamma\left(t^{2} \bar{t}-\bar{t}^{2} t\right)
$$

as $\left(\xi_{1}-\xi_{2}\right) \beta \gamma+\alpha\left(\gamma^{2}-\beta^{2}\right)=\left(\xi_{1}-\xi_{2}\right)(i \beta) \overline{i \gamma}+\alpha\left(|i \gamma|^{2}-|i \beta|^{2}\right)=0$ by Eq.(3.13). If $\xi_{1} \neq \xi_{2}$, then $\left(\xi_{1}-\xi_{2}\right) \beta \gamma \neq 0$ and it is clear that we can choose $t, s, p$ with $t s \bar{p} \notin \mathbb{R}$ so that $\operatorname{det}\left(\left[B, F_{t, s, p}\right]\right) \neq 0$. Thus we get $h(B)=h\left(F_{t, s, p}\right)=h(A)$. If $\xi_{1}=\xi_{2}$, then we must have $\xi_{2} \neq \xi_{3}$. Now consider the term of $\operatorname{det}\left(\left[B, F_{t, s, p}\right]\right)$ that only contain $s$, which is

$$
i\left(\xi_{2}-\xi_{3}\right) \alpha \gamma\left(s^{2} \bar{s}-\bar{s}^{2} s\right)+i \beta\left(\alpha^{2}-\gamma^{2}\right)\left(s^{2} \bar{s}+\bar{s}^{2} s\right)=2 i\left(\xi_{2}-\xi_{3}\right) \alpha \gamma s^{2} \bar{s}
$$

since $\left(\xi_{2}-\xi_{3}\right) \alpha \bar{i} \gamma+(i \beta)\left(|\alpha|^{2}-|i \gamma|^{2}\right)=0$ by Eq.(3.13). Clearly $\left(\xi_{2}-\xi_{3}\right) \alpha \gamma \neq 0$ implies that there are $t, s, p$ with $t s \bar{p} \notin \mathbb{R}$ so that $\operatorname{det}\left(\left[B, F_{t, s, p}\right]\right) \neq 0$. It follows that $h(B)=h\left(F_{t, s, p}\right)=h(A)$.

The cases $1^{\circ}, 3^{\circ}$ and $4^{\circ}$ are dealt with similarly. This completes the proof of the Claim 4.

Claim 5. For any $A, B \in \mathcal{B}_{s}(H) \backslash \mathcal{D}$, we have $h(A)=h(B)$.

By Lemma 3.2, there exist $E, F \in \mathcal{B}_{s}(H) \backslash \mathcal{D}$ of rank not greater than 2 such that $W(A E-$ $E F) \neq-W(A E-E F)$ and $W(B F-F B) \neq-W(B F-F B)$. Thus we get $h(A)=h(E)$ and $h(B)=h(F)$. However, by Claims 2-4, we always have $h(E)=h(F)$. Hence $h(A)=h(B)$.

Finally, let $\mathcal{S}=\{S \in \mathcal{D}: h(S) \neq h(A)$ for $A \notin \mathcal{D}\}$. Then it is clear that the theorem holds. 


\section{The CASe When $\operatorname{dim} H=2$}

In this last section we consider the problem for the case when $\operatorname{dim} H=2$. As we will see the situation for the two dimensional case is much different from that for the case of dimension $\geq 3$.

As $\operatorname{dim} H=2$, we can identify $\mathcal{B}_{s}(H)$ as $\mathbf{H}_{2}=\mathbf{H}_{2}(\mathbb{C})$, the set of all $2 \times 2$ Hermitian matrices over $\mathbb{C}$.

The following is our result and the surjectivity assumption on $\Phi$ is not needed.

Theorem 4.1. Let $\Phi: \mathbf{H}_{2}(\mathbb{C}) \rightarrow \mathbf{H}_{2}(\mathbb{C})$ be a map. The following statements are equivalent.

(1) $\sigma([\Phi(A), \Phi(B)])=\sigma([A, B])$ for any $A, B \in \mathbf{H}_{2}(\mathbb{C})$.

(2) $W([\Phi(A), \Phi(B)])=W([A, B])$ for any $A, B \in \mathbf{H}_{2}(\mathbb{C})$.

(3) $w([\Phi(A), \Phi(B)])=w([A, B])$ for any $A, B \in \mathbf{H}_{2}(\mathbb{C})$.

(4) There exist a unitary matrix $U \in M_{2}(\mathbb{C})$, a sign function $h: \mathbf{H}_{2} \rightarrow\{-1,1\}$ and a functional $f: \mathbf{H}_{2}(\mathbb{C}) \rightarrow \mathbb{R}$ such that one of the following holds:

$\left(1^{\circ}\right) \Phi(A)=h(A) U A U^{*}+f(A) I$ for all $A \in \mathbf{H}_{2}$;

$\left(2^{\circ}\right) \Phi(A)=h(A) U A^{t} U^{*}+f(A) I$ for all $A \in \mathbf{H}_{2}$;

$\left(3^{\circ}\right) \Phi(A)=h(A) U \Psi(A) U^{*}+f(A) I$ for all $A \in \mathbf{H}_{2}$;

$\left(4^{\circ}\right) \Phi(A)=h(A) U \Psi(A)^{t} U^{*}+f(A) I$ for all $A \in \mathbf{H}_{2}$.

Where, with $A=\left(\begin{array}{cc}a & c+i d \\ c-i d & b\end{array}\right), \Psi(A)=\left(\begin{array}{cc}a & -c+i d \\ -c-i d & b\end{array}\right)$.

Proof. It is clear that $(4) \Rightarrow(1) \Leftrightarrow(2) \Leftrightarrow(3)$.

$(3) \Rightarrow(4)$. Assume $\Phi: \mathbf{H}_{2} \rightarrow \mathbf{H}_{2}$ preserves the numerical radius of Lie product.

We may modify the functional $f(A)$ in the map $\Phi$ so that $\Phi(A)$ has trace 0 for all $A \in \mathbf{H}_{2}(\mathbb{C})$. Then we can focus on the set $\mathbf{H}_{2}^{0}$ of trace zero matrices in $\mathbf{H}_{2}(\mathbb{C})$.

Now, suppose (1) holds.

Consider the Hermitian matrices

$$
X=\frac{1}{\sqrt{2}}\left(\begin{array}{cc}
0 & 1 \\
1 & 0
\end{array}\right), \quad Y=\frac{1}{\sqrt{2}}\left(\begin{array}{cc}
0 & -i \\
i & 0
\end{array}\right), \quad Z=\frac{1}{\sqrt{2}}\left(\begin{array}{cc}
1 & 0 \\
0 & -1
\end{array}\right) .
$$

Then the following holds:

(1) $\{X, Y, Z\}$ is an orthonormal basis for $M_{2}^{0}$ using the inner product $\langle A, B\rangle=\operatorname{tr}\left(A B^{*}\right)$, where $M_{2}^{0}$ is the set of trace zero $2 \times 2$ matrices.

(2) $A=a_{1} X+a_{2} Y+a_{3} Z \in \mathbf{H}_{2}^{0}$ if and only if $\left(a_{1}, a_{2}, a_{3}\right)^{t} \in \mathbb{R}^{3}$.

(3) $X Y=\frac{i}{\sqrt{2}} Z=-Y X, \quad Y Z=\frac{i}{\sqrt{2}} X=-Z Y, \quad Z X=\frac{i}{\sqrt{2}} Y=-X Z$.

(4) $W([X, Y])=W([Y, Z])=W([Z, X])=i[-1,1]$.

(5) If $A=a_{1} X+a_{2} Y+a_{3} Z$ and $B=b_{1} X+b_{2} Y+b_{3} Z$ in $M_{2}^{0}$, then

$$
[A, B]=\sqrt{2} i\left(c_{1} X+c_{2} Y+c_{3} Z\right),
$$

where

$$
c_{1}=a_{2} b_{3}-a_{3} b_{2}, \quad c_{2}=-\left(a_{1} b_{3}-a_{3} b_{1}\right), \quad c_{3}=a_{1} b_{2}-a_{2} b_{1} .
$$

In other words, $\left(c_{1}, c_{2}, c_{3}\right)^{t}=\left(a_{1}, a_{2}, a_{3}\right)^{t} \times\left(b_{1}, b_{2}, b_{3}\right)^{t}$, the cross product in $\mathbb{C}^{3}$. 
(6) Every unitary similarity map $a_{1} X+a_{2} Y+a_{3} Z=A \mapsto U A U^{*}=b_{1} X+b_{2} Y+b_{3} Z$ on $M_{2}^{0}$ corresponds to a real special orthogonal transformation $T \in M_{3}(\mathbb{C})$ such that $T\left(a_{1}, a_{2}, a_{3}\right)^{t}=$ $\left(b_{1}, b_{2}, b_{3}\right)^{t}$.

Claim 1. There exist a unitary $U \in M_{2}(\mathbb{C})$ such that

$$
\Phi(A)=\varepsilon_{A} U A U^{*}
$$

for all $A \in\{X, Y, Z\}$, where $\varepsilon_{A} \in\{-1,1\}$.

Assume that the image of $X, Y, Z$ are respectively

$$
X_{1}=a_{11} X+a_{21} Y+a_{31} Z, Y_{1}=a_{12} X+a_{22} Y+a_{32} Z, Z_{1}=a_{13} X+a_{23} Y+a_{33} Z .
$$

Then $a_{p q}$ s are real numbers. Let $T=\left(a_{p q}\right) \in M_{3}(\mathbb{R})$. We will show that $T$ is a real orthogonal matrix. Thus $\Phi$ has the form in Claim 6.

Note that the hypothesis and conclusion will not be affected by changing $T$ to $P T Q$ for any real orthogonal matrices $P, Q \in M_{3}(\mathbb{C})$. It just corresponds to changing $\Phi$ to a map of the form

$$
A \mapsto \varepsilon_{P} U_{P} \Phi\left(\varepsilon_{Q} U_{Q} A U_{Q}^{*}\right) U_{P}^{*}
$$

for some unitary $U_{P}, U_{Q} \in M_{2}(\mathbb{C})$ and $\varepsilon_{P}, \varepsilon_{Q} \in\{1,-1\}$ depending on $P$ and $Q$.

By the singular value decomposition of real matrices, let $P, Q$ be real orthogonal such that $P T Q=\operatorname{diag}\left(s_{1}, s_{2}, s_{3}\right)$ with $s_{1} \geq s_{2} \geq s_{3} \geq 0$. Now, replace $T$ by $P T Q$ so that $T=\operatorname{diag}\left(s_{1}, s_{2}, s_{3}\right)$. Thus there exists a real orthogonal matrix $U \in M_{2}(\mathbb{C})$ such that

$$
\Phi(X)=s_{1} U X U^{*}, \Phi(Y)=s_{2} U Y U^{*}, \Phi(Z)=s_{3} U Z U^{*} .
$$

It follows that

$$
1=w(X Y-Y X)=w(\Phi(X) \Phi(Y)-\Phi(Y) \Phi(X))=\left|s_{1} s_{2}\right| w(X Y-Y X)=\left|s_{1} s_{2}\right| .
$$

Similarly, one gets $\left|s_{1} s_{3}\right|=\left|s_{2} s_{3}\right|=1$ and hence $s_{1}, s_{2}, s_{3} \in\{-1,1\}$. Thus the Claim is true.

Without loss of generality in the sequel we assume $U=I_{2}$. Note that, for any sign function $h: \mathbf{H}_{2} \rightarrow\{-1,1\}$, the map $\Psi$ defined by $\Psi(A)=h(A) \Phi(A)$ still preserves the numerical radius of Lie product. So, multiplied by a suitable sign function if necessary, we may assume that

$$
\Phi(C)=C
$$

for every $C \in\{X, Y, Z\}$.

Claim 2. There are sign functions $\varepsilon_{1}, \varepsilon_{2}, \varepsilon_{3}: \mathbf{H}_{2} \rightarrow\{-1,1\}$ and a functional $f: \mathbf{H}_{2} \rightarrow \mathbb{R}$ such that, for any $A \in \mathbf{H}_{2}$ with $A=\left(\begin{array}{cc}a & c+i d \\ c-i d & b\end{array}\right)$, we have

$$
\Phi(A)=\left(\begin{array}{cc}
\varepsilon_{1}(A) a & \varepsilon_{2}(A) c+i \varepsilon_{3}(A) d \\
\varepsilon_{2}(A) c+i \varepsilon_{3}(A) d & \varepsilon_{1}(A) b
\end{array}\right)+f(A) I_{2}
$$

Write $A=\left(\begin{array}{cc}a & c+i d \\ c-i d & b\end{array}\right)$ and $\Phi(A)=\left(\begin{array}{cc}x & w+i v \\ w-i v & y\end{array}\right)$, where $a, b, c, d, x, y, w, v$ are real numbers. Note that, for any $E, F \in \mathbf{H}_{2}, w(E F-F E)=\delta$ if and only if the spectrum 
$\sigma(E F-F E)=i[-\delta, \delta]$. Thus $w(A C-C A)=w(B C-C B)$ if and only if $\sigma(A C-C A)=$ $\sigma(B C-C B)$. As

$$
\begin{array}{lll}
\sqrt{2}(A X-X A)=\left(\begin{array}{cc}
i 2 d & a-b \\
b-a & -i 2 d
\end{array}\right), & \sqrt{2}(\Phi(A) X-X \Phi(A))=\left(\begin{array}{cc}
2 i v & x-y \\
y-x & -2 i v
\end{array}\right) \\
\sqrt{2}(A Y-Y A)=i\left(\begin{array}{cc}
2 c & b-a \\
b-a & -2 c
\end{array}\right), & \sqrt{2}(\Phi(A) Y-Y \Phi(A))=i\left(\begin{array}{cc}
2 w & y-x \\
y-x & -2 w
\end{array}\right) ; \\
\sqrt{2}(A Z-Z A)=2\left(\begin{array}{cc}
0 & -c-i d \\
c-i d & 0
\end{array}\right), & \sqrt{2}(\Phi(A) Z-Z \Phi(A))=2\left(\begin{array}{cc}
0 & -w-i v \\
w-i v & 0
\end{array}\right),
\end{array}
$$

we must have

$$
\left\{\begin{array}{l}
4 v^{2}+(x-y)^{2}=4 d^{2}+(a-b)^{2} \\
4 w^{2}-(x-y)^{2}=4 c^{2}-(a-b)^{2} \\
w^{2}+v^{2}=c^{2}+d^{2}
\end{array}\right.
$$

It follows that, the map $\Phi$ sends $\left(\begin{array}{ll}a & 0 \\ 0 & b\end{array}\right)=\left(\begin{array}{cc}\frac{a-b}{2} & 0 \\ 0 & -\frac{a-b}{2}\end{array}\right)+\frac{a+b}{2} I_{2}$ to $\left(\begin{array}{cc}\varepsilon_{1} \frac{a-b}{2} & 0 \\ 0 & -\varepsilon_{1} \frac{a-b}{2}\end{array}\right)+$ $\lambda^{\prime} I_{2}=\left(\begin{array}{cc}\varepsilon_{1} a & 0 \\ 0 & \varepsilon_{1} b\end{array}\right)+\lambda I_{2}$, and sends $\left(\begin{array}{cc}0 & c+i d \\ c-i d & 0\end{array}\right)$ to $\left(\begin{array}{cc}0 & \varepsilon_{2} c+i \varepsilon_{3} d \\ \varepsilon_{2} c-i \varepsilon_{3} d & 0\end{array}\right)+$ $\lambda_{2} I_{2}$ for some scalars $\varepsilon_{1}, \varepsilon_{2}, \varepsilon_{3} \in\{-1,1\}$.

To sum up,

$$
\Phi\left(\left(\begin{array}{ll}
a & 0 \\
0 & b
\end{array}\right)+\mathbb{R} I_{2}\right) \subseteq \varepsilon_{1}\left(\begin{array}{ll}
a & 0 \\
0 & b
\end{array}\right)+\mathbb{R} I_{2}
$$

and

$$
\Phi\left(\left(\begin{array}{cc}
0 & c+i d \\
c-i d & 0
\end{array}\right)+\mathbb{R} I_{2}\right) \subseteq\left(\begin{array}{cc}
0 & \varepsilon_{2} c+i \varepsilon_{3} d \\
\varepsilon_{2} c-i \varepsilon_{3} d & 0
\end{array}\right)+\mathbb{R} I_{2}
$$

where $\varepsilon_{1}, \varepsilon_{2}, \varepsilon_{3} \in\{-1,1\}$ depending on $a, c, d$.

To consider the general $A=\left(\begin{array}{cc}a & c+i d \\ c-i d & b\end{array}\right)$, for any unit vector $x \in \mathbb{C}^{2}$, take unit vector $y \perp x$. Then, with respect to the orthonormal base $\{x, y\}$, one can take

$$
X^{\prime}=\frac{1}{\sqrt{2}}(x \otimes y+y \otimes x), Y^{\prime}=\frac{1}{\sqrt{2}} i(-x \otimes y+y \otimes x), Z^{\prime}=\frac{1}{\sqrt{2}}(x \otimes x-y \otimes y) .
$$

Repeat the argument as in Claim 1 and the above one achieves that, there exists a unitary matrix $U_{x}$ such that

$$
\Phi\left(a x \otimes x+b y \otimes y+\mathbb{R} I_{2}\right) \subseteq \varepsilon_{1}(x, a, b)\left(a U_{x} x \otimes U_{x} x+b U_{x} y \otimes U_{x} y\right)+\mathbb{R} I_{2}
$$

for any $a, b \in \mathbb{R}$ and

$$
\begin{array}{ll} 
& \Phi\left((c+i d) x \otimes y+(c-i d) y \otimes x+\mathbb{R} I_{2}\right) \\
\subseteq \quad & \left(\varepsilon_{2}(x, c, d) c+i \varepsilon_{3}(x, c, d) d\right) u_{x} x \otimes U_{x} y \\
& +\left(\varepsilon_{2}(x, c, d) c-i \varepsilon_{3}(x, c, d) d\right) U_{x} y \otimes U_{x} x+\mathbb{R} I_{2},
\end{array}
$$


where $\left.\varepsilon_{1}(x, a, b), \varepsilon_{2}(x, c, d), \varepsilon_{3}(x, c, d)\right) \in\{-1,1\}$. Particularly, by Eq.(4.3), without loss of generality we may assume that

$$
\sigma(\Phi(A))=\sigma(A)
$$

for all $A \in \mathbf{H}_{2}$. It follows that, if $b=-a$, that is, if $A \in \mathbf{H}_{2}^{0}$, then we have

$$
x^{2}+w^{2}+v^{2}=a^{2}+c^{2}+d^{2},
$$

which, together with Eq.(4.2), gives

$$
x^{2}=a^{2}, w^{2}=c^{2}, v^{2}=d^{2} .
$$

Therefore, we still have

$$
x=\varepsilon_{1} a, w=\varepsilon_{2} c, v=\varepsilon_{3} d
$$

for some $\varepsilon_{1}, \varepsilon_{2}, \varepsilon_{3} \in\{-1,1\}$. Now, it is easily checked that

$$
\Phi\left(\left(\begin{array}{cc}
a & c+i d \\
c-i d & b
\end{array}\right)\right) \in\left(\begin{array}{cc}
\varepsilon_{1} a & \varepsilon_{2} c+i \varepsilon_{3} d \\
\varepsilon_{2} c+i \varepsilon_{3} d & \varepsilon_{1} b
\end{array}\right)+\mathbb{R} I_{2}
$$

for some $\varepsilon_{1}, \varepsilon_{2}, \varepsilon_{3} \in\{-1,1\}$, and the Claim 2 is true.

Replacing $\Phi$ by $\varepsilon_{3}(\Phi-f)$ if necessary, by Claim 2 , we may assume that $\varepsilon_{3} \equiv 1$ and

$$
\Phi(A)=\Phi\left(\left(\begin{array}{cc}
a & c+i d \\
c-i d & b
\end{array}\right)\right)=\left(\begin{array}{cc}
\varepsilon_{1}(A) a & \varepsilon_{2}(A) c+i d \\
\varepsilon_{2}(A) c-i d & \varepsilon_{1}(A) b
\end{array}\right)
$$

for every $A \in \mathbf{H}_{2}$.

To determine the sign functions $\varepsilon_{1}, \varepsilon_{2}$ it is enough to consider their behaviors on $\mathbf{H}_{2}^{0}$.

Let $\mathcal{M}=\left\{A \in \mathbf{H}_{2}^{0}: \varepsilon_{1}(A)=\varepsilon_{2}(A)\right\}$ and $\mathcal{N}=\left\{B \in \mathbf{H}_{2}^{0}: \varepsilon_{1}(B) \neq \varepsilon_{2}(B)\right\}$.

Claim 2. Either $\mathcal{M}=\mathbf{H}_{2}^{0}$ or $\mathcal{N}=\mathbf{H}_{2}^{0}$.

For any $A=\left(\begin{array}{cc}a & c+i d \\ c-i d & -a\end{array}\right), B=\left(\begin{array}{cc}b & e+i f \\ e-i f & -b\end{array}\right) \in \mathbf{H}_{2}^{0}$, writing $\varepsilon_{1}=\varepsilon_{j}(A)$ and $\eta_{j}=\varepsilon_{j}(B)$, a simple computation shows that

$$
A B-B A=2\left(\begin{array}{cc}
i(d e-c f) & a e-b c+i(a f-b d) \\
-a e+b c+i(a f-b d) & -i(d e-c f)
\end{array}\right)
$$

and

$$
\begin{aligned}
& \Phi(A) \Phi(B)-\Phi(B) \Phi(A) \\
= & 2\left(\begin{array}{cc}
i\left(\eta_{2} d e-\varepsilon_{2} c f\right) & \varepsilon_{1} \eta_{2} a e-\varepsilon_{2} \eta_{1} b c+i\left(\varepsilon_{1} a f-\eta_{1} b d\right) \\
-\varepsilon_{1} \eta_{2} a e+\varepsilon_{2} \eta_{1} b c+i\left(\varepsilon_{1} a f-\eta_{1} b d\right) & -i\left(\eta_{2} d e-\varepsilon_{2} c f\right)
\end{array}\right) .
\end{aligned}
$$

Since $w(\Phi(A) \Phi(B)-\Phi(B) \Phi(A))=w(A B-B A)$, one gets

$$
\begin{aligned}
& \left(\eta_{2} d e-\varepsilon_{2} c f\right)^{2}+\left(\varepsilon_{1} \eta_{2} a e-\varepsilon_{2} \eta_{1} b c\right)^{2}+\left(\varepsilon_{1} a f-\eta_{1} b d\right)^{2} \\
= & (d e-c f)^{2}+(a e-b c)^{2}+(a f-b d)^{2},
\end{aligned}
$$

that is,

$$
\begin{aligned}
& d^{2} e^{2}+c^{2} f^{2}+a^{2} f^{2}+b^{2} d^{2}-2 d f\left(\varepsilon_{2} \eta_{2} c e+\varepsilon_{1} \eta_{1} a b\right)-2 \varepsilon_{1} \varepsilon_{2} \eta_{1} \eta_{2} a b c e \\
= & d^{2} e^{2}+c^{2} f^{2}+a^{2} f^{2}+b^{2} d^{2}-2 d f(c e+a b)-2 a b c e,
\end{aligned}
$$


which gives

$$
d f\left(\varepsilon_{2} \eta_{2} c e+\varepsilon_{1} \eta_{1} a b\right)+\varepsilon_{1} \varepsilon_{2} \eta_{1} \eta_{2} a b c e=d f(c e+a b)+a b c e .
$$

Assume that both $\mathcal{M}$ and $\mathcal{N}$ are not empty. Obviously, we can require $\varepsilon_{1}(A)=\varepsilon_{2}(A)$ if $a c=0$. So, $\mathcal{Q}=\left\{A=\left(\begin{array}{cc}a & c+i d \\ c-i d & -a\end{array}\right) \in \mathbf{H}_{2}^{0}: a c=0\right\} \subseteq \mathcal{M} \cap \mathcal{N}$. If one of $\mathcal{M}$ and $\mathcal{N}$ is a subset of $\mathcal{Q}$, then the claim is true. Assume that none of $\mathcal{M}$ and $\mathcal{N}$ is a subset of $\mathcal{Q}$. We show that this leads to a contradiction.

Let $\mathcal{N}_{1}=\mathcal{N} \backslash \mathcal{Q}$. Then $\mathcal{N}_{1}$ is not a empty set, and $B=\left(\begin{array}{cc}b & e+i f \\ e-i f & -b\end{array}\right) \in \mathcal{N}_{1}$ implies that $b e \neq 0$. For any $A=\left(\begin{array}{cc}a & c+i d \\ c-i d & -a\end{array}\right) \in \mathcal{M}, B=\left(\begin{array}{cc}b & e+i f \\ e-i f & -b\end{array}\right) \in \mathcal{N}$, since $\varepsilon_{2}=\varepsilon_{1}=\varepsilon \in\{-1,1\}$ and $\eta_{2}=-\eta_{1}=\eta \in\{-1,1\}$, Eq.(4.9) gives

$$
d f \varepsilon \eta(a b-c e)-a b c e=d f(a b+c e)+a b c e .
$$

Thus,

if $\varepsilon \eta=1$, one gets $d f c e=-a b c e$, that is, $d f c=-a b c$ as $b e \neq 0$;

if $\varepsilon \eta=-1$, one gets $d f a b=-a b c e$, that is, $a d f=-a c e$ as $b e \neq 0$.

Assume that $f=0$ for some $\left(\begin{array}{cc}b & e+i f \\ e-i f & -b\end{array}\right) \in \mathcal{N}_{1}$; then we must have $a c=0$ for all $\left(\begin{array}{cc}a & c+i d \\ c-i d & -a\end{array}\right) \in \mathcal{M}$, which is a contradiction. Thus, for all $B=\left(\begin{array}{cc}b & e+i f \\ e-i f & -b\end{array}\right) \in$ $\mathcal{N}_{1}$, we have bef $\neq 0$. Hence, for any $A \in \mathcal{M}, B \in \mathcal{N}_{1}$,

$$
\begin{gathered}
\varepsilon(A) \varepsilon_{1}(B)=1 \text { and } c \neq 0 \Rightarrow d f=-a b \\
\varepsilon(A) \varepsilon_{1}(B)=-1 \text { and } a \neq 0 \Rightarrow d f=-c e .
\end{gathered}
$$

Fix some $A, B$ as above. Take $D=\left(\begin{array}{cc}x & y+i z \\ y-i z & -x\end{array}\right) \in \mathbf{H}_{2}^{0}$ so that $x y z \neq 0, \frac{z}{x} \notin\left\{\frac{d}{a}, \frac{f}{b}\right\}$ and $\frac{z}{y} \notin\left\{\frac{d}{c}, \frac{f}{e}\right\}$. Then it is easily checked that $D \neq \mathcal{M} \cup \mathcal{N}=\mathbf{H}_{2}^{0}$, a contradiction. So, we must have $\mathcal{M}=\mathbf{H}_{2}^{0}$ or $\mathcal{N}=\mathbf{H}_{2}^{0}$.

Claim 3. If $\mathcal{M}=\mathbf{H}_{2}^{0}$, then $\Phi$ has the form $\left(1^{\circ}\right)$ or $\left(2^{\circ}\right)$.

Let $\mathcal{M}_{+}=\left\{B \in \mathcal{M}: \varepsilon_{1}(B)=1\right\}$ and $\mathcal{M}_{-}=\left\{B \in \mathcal{M}: \varepsilon_{1}(B)=-1\right\}$. Then $\mathbf{H}_{2}^{0}=\mathcal{M}=$ $\mathcal{M}_{+} \cup \mathcal{M}_{-}$and $\mathcal{M}_{+} \cap \mathcal{M}_{-}=\left\{\left(\begin{array}{cc}0 & \text { if } \\ -i f & 0\end{array}\right): f \in \mathbb{R}\right\}$. It is clear that $\Phi(A)=A$ if $A \in \mathcal{M}_{+}$ and $\Phi(A)=-A^{t}$ if $A \in \mathcal{M}_{-}$.

For any $A=\left(\begin{array}{cc}a & c+i d \\ c-i d & -a\end{array}\right) \in \mathcal{M}_{+}$and $B=\left(\begin{array}{cc}b & e+i f \\ e-i f & -b\end{array}\right) \in \mathcal{M}_{-}$, by Eq.(4.9) we have

$$
d f(a b+c e)=0 .
$$

Assume $d f=0$; then the above equation is always true. If $f=0$, then $B$ is a real matrix and $\Phi(B)=-B^{t}=-B$. Letting $h(B)$ absorb a -1 we may require that $B \in \mathcal{M}_{+}$. Similarly, 
if $d=0$, we may rearrange if necessary so that $A \in \mathcal{M}_{-}$. Hence we may require that one of $\mathcal{M}_{ \pm}$contains no real matrices.

If one of $\mathcal{M}_{ \pm}$consists of real matrices, we already prove that $\Phi$ has the form $\left(1^{\circ}\right)$ or $\left(2^{\circ}\right)$

Assume that $\mathcal{M}_{+}$and $\mathcal{M}_{-}$contain respectively non-real matrices $A$ and $B$; then $d f \neq 0$. It follows that

$$
a b+c e=0 .
$$

If $a b c e \neq 0$, we get

$$
\frac{e}{b}=-\frac{a}{c}
$$

Take $D=\left(\begin{array}{cc}x & y+i z \\ y-i z & -x\end{array}\right) \in \mathbf{H}_{2}^{0}$ with $x y z \neq 0, \frac{y}{x} \notin\left\{\frac{c}{a}, \frac{e}{b}\right\}$. Then either $D \in \mathcal{M}_{+}$ or $D \in \mathcal{M}_{-}$. However, $D \in \mathcal{M}_{+}$implies that $\frac{y}{x}=-\frac{b}{e}=\frac{c}{a}$ and $D \in \mathcal{M}_{-}$implies that $\frac{y}{x}=-\frac{a}{c}=\frac{e}{b}$, contradicting to the choice of $D$. Hence we always have abce $=0$, that is, at lest one of $a, b, c, e$ is zero. Without loss of generality, assume that $a c \neq 0$; then $b e=0$. In fact we have $b=e=0$ since $a b+c e=0$. This forces that $\mathcal{M}_{-}=\left\{\left(\begin{array}{cc}0 & \text { if } \\ -i f & 0\end{array}\right): f \in \mathbb{R}\right\}$. and therefore $\mathcal{M}_{+}=\mathbf{H}_{2}^{0}$. In this case we have $\Phi(A)=A$ for all $A \in \mathbf{H}_{2}^{0}$ and $\Phi$ has the form $\left(1^{\circ}\right)$. If $b e \neq 0$ and $a c=0$, one gets $a=c=0$ and thus

$$
\mathcal{M}_{+} \subseteq \mathcal{R}=\left\{\left(\begin{array}{cc}
u & w+i v \\
w-i v & -u
\end{array}\right): v=0 \text { or } u=w=0\right\} .
$$

So we may require that $\mathcal{M}_{-}=\mathbf{H}_{2}^{0}$ and $\Phi(A)=-A^{t}$ for every $A \in \mathbf{H}_{2}^{0}$, which implies that $\Phi$ has the form $\left(2^{\circ}\right)$. If $a c=b e=0$ for any $A, B$ with $d f \neq 0$, then we get a contradiction that $D=\left(\begin{array}{cc}x & y+i z \\ y-i z & -x\end{array}\right) \in \mathbf{H}_{2}^{0}$ with $x y z \neq 0$ does not in $\mathcal{M}_{+} \cup \mathcal{M}_{-}=\mathbf{H}_{2}^{0}$. This completes the proof of Claim 3.

Claim 4. If $\mathcal{N}=\mathbf{H}_{2}^{0}$, then $\Phi$ has the form $\left(3^{\circ}\right)$ or $\left(4^{\circ}\right)$.

Let $\mathcal{N}_{+}=\left\{B \in \mathcal{N}: \varepsilon_{1}(B)=1\right\}$ and $\mathcal{N}_{-}=\left\{B \in \mathcal{N}: \varepsilon_{1}(B)=-1\right\}$. Then $\mathbf{H}_{2}^{0}=\mathcal{N}=$ $\mathcal{N}_{+} \cup \mathcal{N}_{-}$and still, $\mathcal{N}_{+} \cap \mathcal{N}_{-}=\left\{\left(\begin{array}{cc}0 & \text { if } \\ -i f & 0\end{array}\right): f \in \mathbb{R}\right\}$. Clearly, $\Phi(A)=\Psi(A)$ if $A \in \mathcal{N}_{+}$ and $\Phi(A)=-\Psi(A)^{t}$ if $A \in \mathcal{N}_{-}$.

Note that, for any $B_{1}, B_{2} \in \mathcal{N}_{+}$or $B_{1}, B_{2} \in \mathcal{N}_{-}$we have $w\left(\left[B_{1}, B_{2}\right]\right)=w\left(\left[\Phi\left(B_{1}\right), \Phi\left(B_{2}\right)\right]\right)$ by Eq.(4.9). Also, if $B$ is real, then $\Phi(B)=-B$. Thus, with no loss of generality we may assume that all real matrices are contained in $\mathcal{N}_{+}$.

For any $A=\left(\begin{array}{cc}a & c+i d \\ c-i d & -a\end{array}\right) \in \mathcal{N}_{+}$and $B=\left(\begin{array}{cc}b & e+i f \\ e-i f & -b\end{array}\right) \in \mathcal{N}_{-}$, by Eq.(4.9) we still have

$$
d f(a b+c e)=0 .
$$

If for any $A \in \mathcal{N}_{+}$and $B \in \mathcal{N}_{-}$we always have $d f=0$ whenever $(a, c) \neq(0,0)$, then we must have $d=0$ for any $A \in \mathcal{N}_{+}$, which means that $\mathcal{N}_{+} \subseteq \mathcal{R}$. It is easily checked in this case that $\Phi$ has the form $\left(3^{\circ}\right)$. So, we may assume that $d f \neq 0$ for some $A$ with $(a, c) \neq(0,0)$ and $B$. It 
follows that $a b+c e=0$. The same reason as that in Claim 3 reveals that abce $\neq 0$ will lead to a contradiction. Thus we must have $a b c e=0$. Since there exists $A \in \mathcal{N}_{+}$with acd $\neq 0$ or $B \in \mathcal{N}_{-}$with bef $\neq 0$, a similar argument as that in Claim 3 shows that the prior case implies that $\mathcal{N}_{-}=\left\{\left(\begin{array}{cc}0 & \text { if } \\ -i f & 0\end{array}\right): f \in \mathbb{R}\right\}$ and hence $\Phi$ has the form $\left(3^{\circ}\right)$; the later case implies that $\mathcal{N}_{+}=\mathcal{R}$ and hence $\Phi$ has the form $\left(4^{\circ}\right)$.

\section{REFERENCES}

[1] Z.-F. Bai, J.-C. Hou, Numerical radius distance preserving maps on $\mathcal{B}(H)$, Proc. Amer. Math. Soc. 132 (2004), 1453-1461.

[2] Z.-F. Bai, J.-C. Hou, Z.-B. Xu, Maps preserving numerical radius on C*-algebras, Studia Math. 162 (2004), 97-104.

[3] J.-T. Chan, Numerical radius preserving operators on $\mathcal{B}(H)$, Proc. Amer. Math. Soc. 123 (1995), 14371439.

[4] J.-T. Chan, Numerical radius preserving operators on $\mathrm{C}^{*}$-algebras, Arch. Marh. (Basel) 70 (1998), $486-488$.

[5] J.-T. Chan, C.-K. Li, N.-S. Sze, Mappings on matrices: Invariance of functional values of matrix products, J. Austral. Math. Soc. (Serie A) 81 (2006), 165-184.

[6] J.-L. Cui, J.-C. Hou, Maps preserving functional values of operator products invariant, Linear Algebra Appl. 428 (2008), 1649-1663.

[7] J.-L. Cui, J.-C. Hou, Linear maps preserving the closure of numerical range on nest algebras with maximal atomic nest, Int. Equ. Oper. Theo. 46 (2003), 253-266.

[8] J.-L. Cui, J.-C. Hou, Non-linear numerical radius isometries on atomic nest algebras and diagonal algebras, J. Funct. Anal. 206 (2004), 414-448.

[9] G. Dolinar, K. He, J.-C. Hou, B. Kuzma, Maps on self-adjoint operators preserving numerical range of Lie products up to a factor, preprint.

[10] M. Dobovisek, B. Kuzma, G. Lešnjak. C.-K. Li and T.Petek, Mappings that preserve pairs of operators with zero triple jordan product, Linear Algebra and its Applications Volume 426, Issues 2-3, 15 October 2007, Pages 255-279.

[11] H.-L. Gau, C.-K. Li, C*-Isomorphisms, Jordan Isomorphisms, and Numerical Range Preserving Maps, Proc. Amer. Math. Soc. 135 (2007), 2907-2914.

[12] K. He, J.-C. Hou, X.-L. Zhang, Maps preserving numerical radius or cross norms of products of self-adjoint operators, Acta Mathematica Sinica-English Series 26 (2010), 1071-1086.

[13] J.-C. Hou, K. He, X.-L. Zhang, Nonlinear maps preserving numerical radius of indefinite skew products of operators, Linear Algebra and its Applications., 430 (2009) 2240-2253.

[14] J.-C. Hou, On operator inequalities and linear combinations of operators, Linear Algebra Appl., 153 (1991), 35-51.

[15] J.-C. Hou, Q.-H. Di, Maps preserving numerical range of operator products, Proc. Amer. Math. Soc. 134 (2006) $1435-1446$.

[16] J.-C. Hou, C.-K. Li, X.-F. Qi, Numerical range of Lie product of operators, preprint

[17] C.-K. Li, N.-S. Sze, Product of Operators and Numerical Range Preserving Maps, Studia Math. 174 (2006), 169-182.

[18] C.-K. Li and N.-K. Tsing, Linear preservers on numerical ranges, numerical radii and unitary similarity invariant norms, Linear and Multilinear Algebra 33 (1992), 63-73. 
[19] C.-K. Li, E. Poon, N.-S. Sze, Preservers for norms of Lie products, Operators and Matrices, 3 (2009), $187-203$.

[20] L. Molnár, P. Šemrl, Nonlinear commutativity preserving maps on self-adjoint operators, Quart. J. Math. 56 (2005), 589-595.

(Jinchuan Hou) College of Mathematics, Taiyuan University of Technology, Taiyuan, 030024, P. R. CHINA

E-mail address, J. Hou: jinchuanhou@aliyun.com

(Kan He) College of Mathematics, Taiyuan University of Technology, Taiyuan, 030024, P. R. CHINA

E-mail address, K. He: hk19830310@163.com 